\title{
Ammonia observations towards molecular and optical outflows
}

\author{
G. Anglada ${ }^{1,2}$, I. Sepúlveda ${ }^{1}$, and J.F. Gómez ${ }^{3,4}$ \\ 1 Departament d'Astronomia i Meteorologia, Universitat de Barcelona, Av. Diagonal 647, E-08028 Barcelona, Spain \\ 2 Instituto de Astronomía, UNAM, Apdo. Postal 70-264, 04510 México DF, Mexico \\ 3 Laboratorio de Astrofísica Espacial y Física Fundamental, INTA, Apdo. Correos 50727, E-28080 Madrid, Spain \\ 4 Instituto de Astrofísica de Andalucía, CSIC, Apdo. Correos 3004, C/ Sancho Panza s/n, E-18080 Granada, Spain
}

Received February 14; accepted May 20, 1996

\begin{abstract}
We observed the $(J, K)=(1,1)$ and $(J, K)$ $=(2,2)$ inversion transitions of the $\mathrm{NH}_{3}$ molecule towards several regions with molecular or optical outflows: RNO 43, HH 83, HH 84, HH 86/87/88, L1641-N, L100, L483, L673, IRAS 20188+3928, L1228, L1048, HHL 73, L1251 (IRAS $22343+7501$ and IRAS $22376+7455)$ and L1262, using the $37 \mathrm{~m}$ radio telescope of the Haystack Observatory. Additionally, we searched for the $6_{16}-5_{23} \mathrm{H}_{2} \mathrm{O}$ maser line towards nine regions, detecting a weak $\mathrm{H}_{2} \mathrm{O}$ maser near IRAS 20188+3928. We detected and mapped $\mathrm{NH}_{3}$ emission in 14 of the 15 regions observed, and we estimated physical parameters for the high density gas. We systematically found that the position of the best candidate for the outflow excitation in each region is very close to an $\mathrm{NH}_{3}$ emission peak. From a statistical study of the data presented in this paper, together with previously published data, we conclude that the $\mathrm{NH}_{3}$ line emission is more intense towards molecular outflow sources than towards sources with only optical outflows. Therefore, molecular outflows appear to be associated with larger amounts of high density gas. This result suggests a possible evolutive scheme in which young objects associated with molecular outflows lose progressively their neighboring high-density gas, weakening both the $\mathrm{NH}_{3}$ emission and the molecular outflow in the process, and making optical jets more easily detectable as the total amount of gas decreases.
\end{abstract}

Key words: ISM: jets and outflows - ISM: molecules masers - stars: formation

\section{Introduction}

The early stages of stellar evolution are dominated by processes involving strong mass loss. The effect of this mass outflow on nearby molecular cloud material is evidenced

Send offprint requests to: G. Anglada principally by the presence, in the radio domain, of molecular outflows and, in the optical domain, by the presence of Herbig-Haro objects and highly collimated jets. Several lines of evidence indicate that molecular outflow is one of the earliest observable phases of the stellar evolution (e.g., Rodríguez 1990). Likewise, Eiroa et al. (1994a) and Persi et al. (1994) concluded that an important fraction of what are thought to be the youngest objects (the socalled Class 0 sources; André et al. 1993) are associated with Herbig-Haro objects, suggesting that not only the molecular outflows but also the optical ones start in the early stages of the star formation process.

One of the remaining open questions regarding the outflow phenomenon is that of the driving mechanism of molecular outflows. There is a growing belief that highly collimated (moving at high velocity; $\gtrsim 100 \mathrm{~km} \mathrm{~s}^{-1}$ ) circumstellar $\left(\sim 10^{4} \mathrm{AU}\right)$ optical jets drive the less collimated (and of lower velocity; $\gtrsim 10 \mathrm{~km} \mathrm{~s}^{-1}$ ) molecular outflows that extend to larger, interstellar scales $\left(\sim 10^{5} \mathrm{AU}\right)$. Detailed models have been developed in this line (see, e.g., Raga et al. 1993 and references therein). In these "unified models", a high velocity, collimated wind (which would correspond to the optically detected $\mathrm{HH}$ objects or jets) drives an envelope of slower, less collimated material (e.g., environmental material set into motion by viscous coupling), which is identified with the molecular outflow. Within this scenario, both optical and molecular outflows would coexist during the pre-main-sequence stages. Despite this coevality, depending on the evolutionary stage of a particular young stellar object, observations could appear dominated by either type of mass loss phenomenon. For the youngest objects, which are still deeply embedded in high density molecular material, circumstellar optical jets are expected to be highly extinguished and hardly detectable, while molecular outflows can be more prominent. As the object evolves, the ambient molecular gas is progressively being swept up by the outflow, and the driving 
jet becomes more easily detectable at optical wavelengths. The decrease of the high density gas near the star is expected to be evidenced through a decrease in the line intensity of high-density tracers, such as the $\mathrm{NH}_{3}$ molecule.

Another important issue in the outflow study is the identification of the outflow exciting sources. These sources are commonly embedded in high-density gas, and located near the position of the emission maximum of high-density tracers like the $\mathrm{NH}_{3}$ lines, as shown by Anglada et al. (1989). This association, at a scale $\lesssim 0.1 \mathrm{pc}$, between the ammonia emission peak and the outflow exciting source does not contradict the fact that the ammonia emission could present a much smaller scale structure near the object (e.g., cavities), as revealed by very high angular resolution observations (see the discussion by Anglada et al. 1995). Thus, single-dish ammonia observations can be an useful tool to help to establish the position of an outflow exciting source, to confirm a given candidate or to discriminate between several candidates.

In order to further investigate these issues, we selected a sample of 15 star-forming regions with signs of outflow activity, and we mapped with the Haystack $37 \mathrm{~m}$ telescope the $\mathrm{NH}_{3}$ emission around the position of the suspected outflow exciting sources. In this paper we present the results of this study. In Sect. 2 we describe the observations, in Sect. 3 we discuss the sources individually, in Sect. 4 we discuss the global results of our study, and in Sect. 5 we give our conclusions.

\section{Observations}

The observations were carried out on February 1990 with the $37 \mathrm{~m}$ radio telescope at Haystack Observatory ${ }^{1}$. We observed the $(J, K)=(1,1)$ and the $(J, K)=(2,2)$ inversion transitions of the ammonia molecule. At the observing frequencies $(23.6944960 \mathrm{GHz}$ and $23.7226320 \mathrm{GHz}$, respectively), the beam size of the telescope is 1.4 , and its beam efficiency at an elevation of $45^{\circ}$ is $\sim 0.35$. We used a dual maser receiver and both polarizations were observed. The spectrometer was a 1024-lag digital autocorrelator with an effective bandwidth of $6.67 \mathrm{MHz}$. The calibration was made with the standard noise-tube method. The observations were made in the position switching mode. All the spectra were corrected for the elevation-dependent gain variations and for atmospheric attenuation. The rms pointing error was estimated to be $\sim 15^{\prime \prime}$ by observing continuum unresolved sources. System temperature ranged from 70 to $150 \mathrm{~K}$. The data were reduced using the CLASS and GREG packages of IRAM. The observed spectra were smoothed, resulting a velocity resolution of $\sim 0.2 \mathrm{~km} \mathrm{~s}^{-1}$.

We searched 15 sources for $\mathrm{NH}_{3}(1,1)$ emission. In all cases, we first made measurements on a five-point grid

\footnotetext{
1 Radio Astronomy at Haystack Observatory of the Northeast Radio Observatory Corporation is supported by the National Science Foundation.
}

centered at the positions given in Table 1, with a full beam separation between points. The $\mathrm{NH}_{3}(1,1)$ line was detected in 14 of these sources. The $\mathrm{NH}_{3}(2,2)$ line was observed in 8 sources, at the positions given in Table 2 , and was detected in 6 of them. Spectra of the $\mathrm{NH}_{3}(1,1)$ and $\mathrm{NH}_{3}(2,2)$ lines obtained at the positions given in Table 2 are shown in Figs. 1 and 2, respectively. In Figs. 4 to 13,15 to 17 , and 20 , we show the $\mathrm{NH}_{3}(1,1)$ maps of the detected sources.

In Table 2 we give $\mathrm{NH}_{3}(1,1)$ and $(2,2)$ line parameters obtained from a multicomponent fit to the observed spectra at the position of the emission peak, using the CLASS package. In Table 3 we list physical parameters of the high-density cores, derived from the ammonia observations following the procedures explained in the footnotes of the table.

Additionally, we searched for the $6_{16}-5_{23} \mathrm{H}_{2} \mathrm{O}$ maser line (at the observing frequency of $22.235080 \mathrm{GHz}$ ) in nine sources. We made five or seven-point maps centered at the positions given in Table 1 . For the water maser observations, we used the same spectrometer with the same bandwidth as for the ammonia observations. We reached a typical sensitivity of $1.5 \mathrm{Jy}(1 \sigma)$ per spectral channel. We detected $\mathrm{H}_{2} \mathrm{O}$ maser emission towards the region associated with the source IRAS $20188+3928$ and the spectrum obtained is shown in Fig. 3. For the other observed sources we do not detect any significant $(>3 \sigma) \mathrm{H}_{2} \mathrm{O}$ emission.

\section{Results for individual sources}

\section{1. $R N O 43$}

RNO 43 (Cohen 1980) is the brightest spot of a chain of Herbig-Haro knots (Jones et al. 1984; Ray 1987; Mundt et al. 1987) extending $\sim 5^{\prime}$ to the northeast of the source IRAS $05295+1247$. This chain is designated as $\mathrm{HH} 243$ in the new catalog of Herbig-Haro objects of Reipurth (1994). Another long $\left(\sim 5^{\prime}\right)$, well collimated chain of Herbig-Haro knots (HH 245) extends to the north of RNO 43. Reipurth (1991) found a large fragmented counter bow-shock (HH 244) to the southwest of the IRAS source, suggesting that HH 243 and $\mathrm{HH} 244$ constitute a bipolar HH flow. Anglada et al. (1992) found several radio continuum sources that could be related to the HH $243, \mathrm{HH}$ 244 and HH 245 chains. The high-velocity CO emission in the region has been mapped by Edwards \& Snell (1984), Cabrit et al. (1988), and Bence et al. (1996). The CO outflow exhibits a complex distribution with several overlapping red and blueshifted lobes, extending on both sides of the IRAS source, and aligned roughly in the north-south direction. The CO lobes located to the north of the IRAS source were initially proposed to constitute two separate outflows (Edwards \& Snell 1984). However, no exciting sources have been found for these proposed outflows. Also, no $\mathrm{NH}_{3}$ emission associated with these northern lobes has been detected (Anglada et al. 1989). On the other hand, 
Table 1. Sources observed in $\mathrm{NH}_{3}$ or $\mathrm{H}_{2} \mathrm{O}$

\begin{tabular}{|c|c|c|c|c|c|c|c|c|c|}
\hline \multirow[t]{2}{*}{ Source } & \multicolumn{2}{|c|}{ Central position } & \multirow{2}{*}{$\begin{array}{l}\text { Outflow } \\
\text { type }^{a}\end{array}$} & \multirow{2}{*}{$\begin{array}{c}\text { Excitation } \\
\text { source }^{b}\end{array}$} & \multirow[t]{2}{*}{ Ref. } & \multirow{2}{*}{$\begin{array}{c}\mathrm{NH}_{3} \\
\text { detected? }\end{array}$} & \multirow{2}{*}{$\begin{array}{c}\mathrm{NH}_{3} \\
\text { sensitivity }^{c} \\
(\mathrm{~K})\end{array}$} & \multirow{2}{*}{$\begin{array}{c}\mathrm{H}_{2} \mathrm{O} \\
\text { detected? }\end{array}$} & \multirow{2}{*}{$\begin{array}{c}\mathrm{H}_{2} \mathrm{O} \\
\text { sensitivity }^{c} \\
(\mathrm{Jy})\end{array}$} \\
\hline & $\alpha(1950)$ & $\delta(1950)$ & & & & & & & \\
\hline IRAS $00213+6530$ & $00^{\mathrm{h}} 21^{\mathrm{m}} 22.0$ & $+65^{\circ} 30^{\prime} 25^{\prime \prime}$ & $\mathrm{CO}$ & IRAS & 1 & - & - & No & 1.6 \\
\hline RNO 43 & $05^{\mathrm{h}} 29^{\mathrm{m}} 32^{\mathrm{s}} .7$ & $+12^{\circ} 47^{\prime} 33^{\prime \prime}$ & $\mathrm{CO}, \mathrm{HH}$ & IRAS & $2,3,4$ & Yes & 0.03 & - & - \\
\hline HH 83 & $05^{\mathrm{h}} 31^{\mathrm{m}} 06^{\mathrm{s}} 3$ & $-06^{\circ} 31^{\prime} 45^{\prime \prime}$ & $\mathrm{CO}, \mathrm{HH}$ & IRAS & 5,6 & Yes & 0.03 & - & - \\
\hline HH 84 & $05^{\mathrm{h}} 31^{\mathrm{m}} 44^{\mathrm{s}} .7$ & $-06^{\circ} 35^{\prime} 16^{\prime \prime}$ & $\mathrm{HH}$ & $\mathrm{H} \alpha ?$ & 5 & Yes & 0.04 & - & - \\
\hline L100 & $17^{\mathrm{h}} 13^{\mathrm{m}} 03^{\mathrm{s}} .9$ & $-20^{\circ} 53^{\prime} 39^{\prime \prime}$ & $\mathrm{CO}$ & IRAS & 8 & Yes & 0.05 & - & - \\
\hline L483 & $18^{\mathrm{h}} 14^{\mathrm{m}} 50.6$ & $-04^{\circ} 40^{\prime} 49^{\prime \prime}$ & $\mathrm{CO}$ & IRAS & 8 & Yes & 0.03 & - & - \\
\hline $\mathrm{L} 673$ & $19^{\mathrm{h}} 18^{\mathrm{m}} 01^{\mathrm{s}} 3$ & $+11^{\circ} 16^{\prime} 27^{\prime \prime}$ & $\mathrm{CO}$ & IRAS & 9 & Yes & 0.04 & - & - \\
\hline IRAS $20188+3928$ & $20^{\mathrm{h}} 18^{\mathrm{m}} 50.7$ & $+39^{\circ} 28^{\prime} 18^{\prime \prime}$ & $\mathrm{CO}$ & IRAS & 10 & Yes & 0.03 & Yes & 0.8 \\
\hline L1228 & $20^{\mathrm{h}} 58^{\mathrm{m}} 14.5$ & $+77^{\circ} 24^{\prime} 05^{\prime \prime}$ & $\mathrm{CO}, \mathrm{HH}$ & IRAS & 11,12 & Yes & 0.01 & No & 1.6 \\
\hline L1048 & $21^{\mathrm{h}} 33^{\mathrm{m}} 24^{\mathrm{s}} .0$ & $+50^{\circ} 39^{\prime} 43^{\prime \prime}$ & $\mathrm{CO}$ & IRAS & 13 & No & 0.05 & - & - \\
\hline
\end{tabular}

${ }^{a} \mathrm{CO}=\mathrm{CO}$ outflow HH $=$ Herbig-Haro outflow; HHL = Herbig-Haro-like object.

${ }^{b}$ IRAS $=$ IRAS point source; $\mathrm{H} \alpha=\mathrm{H} \alpha$ emission star.

${ }^{c} 1-\sigma$ rms in the antenna temperature per spectral channel.

References: (1) Fukui 1989; (2) Edwards \& Snell 1984; (3) Cabrit et al. 1988; (4) Jones et al. 1984; (5) Reipurth 1989; (6) Bally et al. 1993; (7) Fukui et al. 1988; (8) Parker et al. 1988; (9) Armstrong \& Winnewisser 1989; (10) Little et al. 1988; (11) Bally et al. 1995; (12) Haikala \& Laureijs 1989; (13) Smith et al. 1989; (14) Dobashi et al. 1993; (15) Gyulbudaghian et al. 1987; (16) Fukui et al. 1986; (17) Eiroa et al. 1993; (18) Sato \& Fukui 1989; (19) Balázs et al. 1992; (20) Eiroa et al. 1994b; (21) Terebey et al. 1989 .

Cabrit et al. (1988) proposed that the overall high-velocity structure constitutes a single bipolar $\mathrm{CO}$ outflow powered by IRAS $05295+1247$. A CS clump (Cabrit et al. 1988) is associated with the IRAS source. However, the nominal position of the IRAS source appears to be displaced $\sim 1^{\prime}$ east from the center of the clump. Anglada et al. (1992) found a $3.6 \mathrm{~cm}$ VLA source (also detected at $1.3 \mathrm{~mm}$ by Reipurth et al. 1993) which is better centered on the CS cloud. Anglada et al. (1992) suggest that the IRAS catalog position is in error by $\sim 30^{\prime \prime}$ in right ascension, and that the IRAS and the radio continuum sources are tracing the same embedded object, which is the powering source of both the molecular and the HH outflows.

In Fig. 4, we show our $\mathrm{NH}_{3}$ map of this region. We have detected a weak $\mathrm{NH}_{3}$ condensation centered $\sim 1^{\prime}$ to the west of the IRAS source, similar to the CS condensation observed by Cabrit et al. (1988). As the exciting sources of outflows are usually deeply embedded objects, the fact that the high-density gas (traced by the CS and $\mathrm{NH}_{3}$ emission; Cabrit et al. 1988; this paper) is found associated only with the cm-mm-IRAS source, and that no $\mathrm{NH}_{3}$ emission was found associated with the northern $\mathrm{CO}$ lobes near RNO 43 (Anglada et al. 1989), further supports that the radio continuum source represents a deeply embedded young stellar object, and is the most plausible powering source for the molecular and $\mathrm{HH}$ outflows observed in this region, as suggested by Anglada et al. (1992).

\section{2. $\mathrm{HH} 83$}

This HH object lies at the western edge of the L1641 molecular cloud. The optical structure consists of a jet (at least $32^{\prime \prime}$ long), apparently emanating from the embedded infrared source HH83 IRS (IRAS 05311 - 0631; Reipurth 1989), which is associated with a reflection nebula and ending with a bow-shock. The jet is highly collimated, and presents large variations in velocity and physical conditions along its length (Reipurth 1989). There is a faint nebulosity $\sim 44^{\prime \prime}$ southeast of the central infrared source that could be part of the counter bow-shock (Reipurth 1989; Ogura \& Walsch 1991). The estimated bolometric luminosity of HH83 IRS is $8 L_{\odot}$ (Reipurth et al. 1993). This source does not present detectable radio continuum emission at 3.6 and $2 \mathrm{~cm}$ (Rodríguez \& Reipurth 1994). Recently, Bally et al. (1994) have mapped a very low velocity, poorly collimated asymmetric molecular outflow (with the redshifted lobe much larger than the blueshifted one) associated with HH83 IRS. These authors suggest 
Table 2. $\mathrm{NH}_{3}$ line parameters ${ }^{a}$

\begin{tabular}{|c|c|c|c|c|c|c|c|c|}
\hline Source & $\begin{array}{l}\text { Position }^{b} \\
\text { (arcmin) }\end{array}$ & $(J, K)$ & $\begin{array}{c}V_{\mathrm{LSR}^{c}} \\
\left(\mathrm{kms}^{-1}\right)\end{array}$ & $T_{(\mathrm{K})}^{T_{\mathrm{MB}}(\mathrm{m})^{d}}$ & $\begin{array}{c}\Delta V^{e} \\
\left(\mathrm{kms}^{-1}\right)\end{array}$ & $\tau_{\mathrm{m}}^{f}$ & $\begin{array}{c}A \tau_{\mathrm{m}}^{g} \\
(\mathrm{~K})\end{array}$ & $\begin{array}{c}N(J, K)^{h} \\
\left(10^{13} \mathrm{~cm}^{-2}\right)\end{array}$ \\
\hline RNO 43 & $(-1.4,0)$ & $(1,1)$ & $+10.26 \pm 0.03$ & $0.46 \pm 0.06$ & $0.63 \pm 0.07$ & $1.1 \pm 0.3$ & $0.80 \pm 0.08$ & 1.4 \\
\hline HH 83 & $(0,0)$ & $(1,1)$ & $+6.22 \pm 0.05$ & $0.46 \pm 0.06$ & $0.4-0.5$ & $\leq 2^{i}$ & $0.6-0.9^{j}$ & $0.8-1.1^{j}$ \\
\hline HH 84 & $(0,0)$ & $(1,1)$ & $+8.3 \pm 0.2$ & $0.3 \pm 0.1$ & $1.3-1.5$ & $\leq 3.6^{i}$ & $0.3-0.7^{j}$ & $1.4-2.5^{j}$ \\
\hline HH $86 / 87 / 88$ & $(1.4,1.4)$ & $(1,1)$ & $+8.88 \pm 0.03$ & $0.57 \pm 0.06$ & $0.72 \pm 0.05$ & $0.1 \pm 0.1$ & $0.73 \pm 0.04$ & 1.5 \\
\hline L1641-N & $(1.4,-2.8)$ & $(1,1)$ & $+6.94 \pm 0.01$ & $2.7 \pm 0.1$ & $0.92 \pm 0.03$ & $1.9 \pm 0.2$ & $6.0 \pm 0.3$ & 15 \\
\hline L100 & $(0,0)$ & $(1,1)$ & $+1.64 \pm 0.08$ & $0.5 \pm 0.1$ & $0.4-0.5$ & $\leq 3^{i}$ & $0.7-1.3^{j}$ & $0.9-1.5^{j}$ \\
\hline L483 & $\begin{array}{l}(0,0) \\
(0,0)\end{array}$ & $\begin{array}{l}(1,1) \\
(2,2)\end{array}$ & $\begin{array}{c}+5.434 \pm 0.003 \\
+5.41 \pm 0.03\end{array}$ & $\begin{array}{l}4.54 \pm 0.09 \\
0.74 \pm 0.06\end{array}$ & $\begin{array}{l}0.57 \pm 0.01 \\
0.79 \pm 0.06\end{array}$ & $\begin{array}{l}4.7 \pm 0.1 \\
0.2 \pm 0.1\end{array}$ & $\begin{array}{c}19.9 \pm 0.3 \\
0.9 \pm 0.4\end{array}$ & $\begin{array}{l}32 \\
0.9\end{array}$ \\
\hline L673 & $\begin{array}{c}(0,-2.8) \\
(1.4,0)\end{array}$ & $\begin{array}{l}(1,1) \\
(2,2)\end{array}$ & $\begin{array}{c}+6.94 \pm 0.01 \\
-\end{array}$ & $\begin{array}{l}2.1 \pm 0.1 \\
\leq 0.17^{k}\end{array}$ & $\begin{array}{c}0.49 \pm 0.02 \\
-\end{array}$ & $\begin{array}{c}1.7 \pm 0.3 \\
-\end{array}$ & $\begin{array}{c}4.9 \pm 0.4 \\
-\end{array}$ & $\begin{array}{c}6.7 \\
-\end{array}$ \\
\hline IRAS $20188+3928$ & $\begin{array}{l}(0,0) \\
(0,0)\end{array}$ & $\begin{array}{l}(1,1) \\
(2,2)\end{array}$ & $\begin{array}{l}+1.57 \pm 0.01 \\
+1.59 \pm 0.02\end{array}$ & $\begin{array}{l}2.66 \pm 0.09 \\
1.31 \pm 0.06\end{array}$ & $\begin{array}{l}1.58 \pm 0.02 \\
1.83 \pm 0.04\end{array}$ & $\begin{array}{l}1.7 \pm 0.1 \\
0.5 \pm 0.2\end{array}$ & $\begin{array}{l}5.3 \pm 0.1 \\
1.7 \pm 0.3\end{array}$ & $\begin{array}{l}23 \\
4.1\end{array}$ \\
\hline L1228 & $\begin{array}{l}(0,0) \\
(0,0)\end{array}$ & $\begin{array}{l}(1,1) \\
(2,2)\end{array}$ & $\begin{array}{c}-8.088 \pm 0.003 \\
-8.06 \pm 0.03\end{array}$ & $\begin{array}{c}2.71 \pm 0.03 \\
0.49 \pm 0.6\end{array}$ & $\begin{array}{l}0.71 \pm 0.01 \\
0.69 \pm 0.06\end{array}$ & $\begin{array}{c}2.31 \pm 0.06 \\
0.2 \pm 0.2\end{array}$ & $\begin{array}{l}7.0 \pm 0.1 \\
0.6 \pm 0.1\end{array}$ & $\begin{array}{l}14 \\
0.5\end{array}$ \\
\hline HHL 73 & $\begin{array}{l}(-2.8,7) \\
(-2.8,7)\end{array}$ & $\begin{array}{l}(1,1) \\
(2,2)\end{array}$ & $\begin{array}{l}+4.20 \pm 0.01 \\
+4.26 \pm 0.06\end{array}$ & $\begin{array}{l}1.66 \pm 0.09 \\
0.31 \pm 0.06\end{array}$ & $\begin{array}{c}0.94 \pm 0.03 \\
0.9 \pm 0.1\end{array}$ & $\begin{array}{l}1.8 \pm 0.2 \\
0.2 \pm 0.3\end{array}$ & $\begin{array}{l}3.5 \pm 0.2 \\
0.4 \pm 0.2\end{array}$ & $\begin{array}{l}9.3 \\
0.4\end{array}$ \\
\hline HHL 73 & $\begin{array}{c}(8.4,2.8) \\
(7,2.8)\end{array}$ & $\begin{array}{l}(1,1) \\
(2,2)\end{array}$ & $\begin{array}{c}+3.74 \pm 0.03 \\
-\end{array}$ & $\begin{array}{l}0.7 \pm 0.1 \\
\leq 0.3^{k}\end{array}$ & $\begin{aligned} 0.67 & \pm 0.07 \\
& -\end{aligned}$ & $\begin{array}{c}3.2 \pm 0.3 \\
-\end{array}$ & $\begin{array}{c}2.1 \pm 0.2 \\
-\end{array}$ & $\begin{array}{c}3.9 \\
-\end{array}$ \\
\hline IRAS $22343+7501$ & $\begin{array}{l}(1.4,1.4) \\
(1.4,1.4)\end{array}$ & $\begin{array}{l}(1,1) \\
(2,2)\end{array}$ & $\begin{array}{l}-4.72 \pm 0.02 \\
-4.68 \pm 0.06\end{array}$ & $\begin{array}{l}1.40 \pm 0.09 \\
0.23 \pm 0.06\end{array}$ & $\begin{array}{c}0.56 \pm 0.04 \\
0.6 \pm 0.1\end{array}$ & $\begin{array}{l}1.7 \pm 0.4 \\
0.1 \pm 0.1\end{array}$ & $\begin{array}{l}3.1 \pm 0.4 \\
0.3 \pm 0.1\end{array}$ & $\begin{array}{l}4.8 \\
0.2\end{array}$ \\
\hline IRAS $22376+7455$ & $\begin{array}{l}(1.4,0) \\
(1.4,0)\end{array}$ & $\begin{array}{l}(1,1) \\
(2,2)\end{array}$ & $\begin{array}{l}-3.53 \pm 0.01 \\
-3.58 \pm 0.04\end{array}$ & $\begin{array}{l}2.29 \pm 0.09 \\
0.37 \pm 0.06\end{array}$ & $\begin{array}{c}0.69 \pm 0.02 \\
0.8 \pm 0.1\end{array}$ & $\begin{array}{l}1.6 \pm 0.2 \\
0.1 \pm 0.2\end{array}$ & $\begin{array}{c}4.9 \pm 0.2 \\
0.43 \pm 0.04\end{array}$ & $\begin{array}{l}9.3 \\
0.5\end{array}$ \\
\hline L1262 & $\begin{array}{c}(-1.4,0) \\
(0,0)\end{array}$ & $\begin{array}{l}(1,1) \\
(2,2)\end{array}$ & $\begin{array}{c}+3.93 \pm 0.01 \\
-\end{array}$ & $\begin{array}{l}1.86 \pm 0.09 \\
\leq 0.17^{k}\end{array}$ & $\begin{array}{c}0.51 \pm 0.02 \\
-\end{array}$ & $\begin{array}{c}3.6 \pm 0.4 \\
-\end{array}$ & $\begin{array}{c}6.6 \pm 0.5 \\
-\end{array}$ & $\begin{array}{c}9.3 \\
-\end{array}$ \\
\hline
\end{tabular}

${ }^{a}$ Obtained from a multicomponent fit to the magnetic hyperfine structure, using the CLASS package.

${ }^{b}$ Position of the emission peak (offset from the central position given in Table 1), where line parameters have been obtained.

${ }^{c}$ Velocity of the line with respect to the local standard of rest.

${ }^{d}$ Main beam brightness temperature of the main line of the transition, obtained from a single Gaussian fit.

${ }^{e}$ Intrinsic line width, obtained taking into account optical depth and hyperfine broadening, but not the spectral resolution of the spectrometer.

${ }^{f}$ Optical depth of the main line. For the $(1,1)$ transition, derived from the relative intensities of the magnetic hyperfine components; for the $(2,2)$ transition, derived from the ratio of the $(1,1)$ and $(2,2)$ antenna temperatures and the optical depth of the $(1,1)$ line, assuming the same excitation temperature for both transitions.

${ }^{g}$ Derived from the transfer equation, where $A=f\left[J\left(T_{\mathrm{ex}}\right)-J\left(T_{\mathrm{bg}}\right)\right]$ is the "amplitude" (Pauls et al. 1983), $f$ is the filling factor, $T_{\text {ex }}$ the excitation temperature of the transition, $T_{\mathrm{bg}}$ the background radiation temperature and $J(T)$ the intensity in units of temperature. Note that $A \simeq f T_{\mathrm{ex}}$, for $T_{\mathrm{ex}} \gg T_{\mathrm{bg}}$.

${ }^{h}$ Beam-averaged column density for the rotational level $(J, K)$, derived from $\left[N(J, K) / \mathrm{cm}^{-2}\right]=C(J, K)\left[A \tau_{\mathrm{m}} / \mathrm{K}\right]\left[\Delta V / \mathrm{km} \mathrm{s}^{-1}\right]$, with $C(1,1)=2.7810^{13}$ and $C(2,2)=1.3110^{13}$ (e.g., Ungerechts et al. 1986).

${ }^{i}$ Obtained adopting a $3 \sigma$ upper limit for the intensity of the satellite lines.

${ }^{j}$ The highest value is obtained with the upper limit of $\tau_{\mathrm{m}}$, and the lowest value is obtained assuming optically thin emission.

${ }^{k} 3 \sigma$ upper limit. 
Table 3. Physical parameters of the $\mathrm{NH}_{3}$ condensations

\begin{tabular}{|c|c|c|c|c|c|c|c|c|c|}
\hline \multirow[t]{2}{*}{ Source } & \multirow{2}{*}{$\begin{array}{r}\text { Distance } \\
(\mathrm{pc})\end{array}$} & \multirow[t]{2}{*}{ Ref. } & \multicolumn{2}{|c|}{ Size $^{a}$} & \multirow{2}{*}{$\begin{array}{c}T_{\text {rot }}^{b} \\
(\mathrm{~K})\end{array}$} & \multirow{2}{*}{$\begin{array}{c}N\left(\mathrm{H}_{2}\right)^{c} \\
\left(10^{22} \mathrm{~cm}^{-2}\right)\end{array}$} & \multirow{2}{*}{$\begin{array}{c}M^{d} \\
\left(M_{\odot}\right)\end{array}$} & \multirow{2}{*}{$\begin{array}{l}M_{\text {vir }}{ }^{e} \\
\left(M_{\odot}\right)\end{array}$} & \multirow{2}{*}{$\begin{array}{c}n\left(\mathrm{H}_{2}\right)^{\mathrm{f}} \\
\left(10^{3} \mathrm{~cm}^{-3}\right)\end{array}$} \\
\hline & & & (arcmin) & (pc) & & & & & \\
\hline RNO 43 & 400 & 1 & $2.6 \times 1.5$ & $0.30 \times 0.18$ & $\sim 15$ & $\sim 0.4$ & $\sim 3$ & 10 & $\sim 1.6$ \\
\hline HH 83 & 470 & 2 & $\gtrsim 2.5 \times 2.3$ & $\gtrsim 0.34 \times 0.31$ & $\sim 10$ & $0.4-0.5$ & $\gtrsim 5$ & $\gtrsim 6$ & $\gtrsim 1.5$ \\
\hline HH 84 & 470 & 2 & $\gtrsim 2.6 \times 2.5$ & $\gtrsim 0.36 \times 0.34$ & $\sim 11$ & $0.5-1$ & $\gtrsim 8$ & $\gtrsim 66$ & $\gtrsim 0.5$ \\
\hline HH $86 / 87 / 88$ & 470 & 2 & $2.4 \times 1.9$ & $0.33 \times 0.26$ & $\sim 19$ & $\sim 0.3$ & $\sim 4$ & 16 & $\sim 24$ \\
\hline L1641-N & 480 & 3 & $2.3 \times 4.5$ & $0.32 \times 0.63$ & $\sim 23$ & $\sim 3.5$ & $\sim 89$ & 40 & $\sim 5.9$ \\
\hline L100 & 225 & 4 & $\gtrsim 2.3 \times 2.4$ & $\gtrsim 0.15 \times 0.16$ & $\sim 12$ & $0.3-0.5$ & $\gtrsim 1$ & $\gtrsim 3$ & $\gtrsim 1.1$ \\
\hline L483 & 200 & 5 & $2.0 \times 1.8$ & $0.12 \times 0.10$ & 10 & 14 & 21 & 4 & 30 \\
\hline L673 & 300 & 6 & $4.7 \times 2.0$ & $0.41 \times 0.18$ & $\leq 12$ & $\geq 2.2$ & $\geq 21$ & 7 & $\geq 11$ \\
\hline IRAS $20188+3928$ & $\leq 4000$ & 7 & $1.7 \times 2.0$ & $\leq 1.99 \times 2.33$ & 18 & 5.5 & $\leq \overline{3} 200$ & $\leq 560$ & 7.3 \\
\hline L1228 & 300 & 8 & $2.9 \times 1.9$ & $0.25 \times 0.17$ & 11 & 5.3 & 28 & 11 & 13 \\
\hline HHL $73(-2.8,7)$ & 900 & 9 & $2.7 \times 2.0$ & $0.71 \times 0.52$ & 12 & 3.2 & 150 & 56 & 6.2 \\
\hline HHL $73(8.4,2.8)$ & 900 & 9 & $3.1 \times 2.4$ & $0.81 \times 0.63$ & $\leq 19$ & $\geq 0.9$ & $\geq 59$ & 34 & $\geq 1.2$ \\
\hline IRAS $22343+7501$ & 300 & 10 & $3.3 \times 2.0$ & $0.29 \times 0.18$ & 12 & 1.7 & 11 & 8 & 5.8 \\
\hline IRAS $22376+7455$ & 300 & 10 & $7.0 \times 1.8$ & $0.61 \times 0.16$ & 12 & 3.1 & 39 & 16 & 11 \\
\hline L1262 & 200 & 11 & $2.8 \times 1.7$ & $0.16 \times 0.10$ & $\leq 9$ & $\geq 4.9$ & $\geq 10$ & 3 & $\geq 8.6$ \\
\hline
\end{tabular}

${ }^{a}$ Major and minor axes of the half-power contour of the $\mathrm{NH}_{3}$ emission.

${ }^{b}$ Rotational temperature, derived from the ratio of $\mathrm{NH}_{3}$ column density in the $(1,1)$ and $(2,2)$ levels (Table 2$)$ for the sources where the $(2,2)$ line was detected. For the sources undetected in the $(2,2)$ line, an upper limit is obtained assuming that the emission is optically thin. For the sources not observed in the $(2,2)$ line, it is assumed that $T_{\text {ex }}(\mathrm{CO})=T_{k}=T_{\text {rot }}(22-11)$, where the CO data are from Cabrit et al. 1988 (RNO 43), Bally et al. 1993 (HH 83), Morgan \& Bally 1991 (HH 84), Maddalena et al. 1986 (HH 86/87/88), Morgan et al. 1991 (L1641-N) and Parker et al. 1988 (L100).

${ }^{c}$ Beam-averaged $\mathrm{H}_{2}$ column density, obtained from the $\mathrm{NH}_{3}$ column density in the rotational states given in Table 2, assuming LTE for the metastable rotational states of $\mathrm{NH}_{3}$ at $T_{k}=T_{R}\left(22-11\right.$ ), and an $\mathrm{NH}_{3}$ abundance $\left[\mathrm{NH}_{3} / \mathrm{H}_{2}\right]=10^{-8}$ (see Anglada et al. 1995 for a discussion on $\mathrm{NH}_{3}$ abundances).

${ }^{d}$ Mass of the condensation, derived from the beam-averaged $\mathrm{H}_{2}$ column density and the observed area.

${ }^{e}$ Virial mass obtained from $\left[M_{\mathrm{vir}} / M_{\odot}\right]=210[R / \mathrm{pc}]\left[\Delta V / \mathrm{km} \mathrm{s}^{-1}\right]^{2}$, where $R$ is the radius of the clump, taken as half the geometrical mean of the two linear sizes, and $\Delta V$ is the intrinsic line width given in Table 2 .

${ }^{f}$ Volume density, derived from the two-level model (Ho \& Townes 1983). $T_{\text {ex }}$ has been obtained from the values of $A$ given in Table 2, assuming $f=1$ (this assumption underestimates $T_{\text {ex }}$ for unresolved sources).

References.-(1) Maddalena \& Morris 1987; (2) Reipurth 1994; (3) Chen et al. 1993; (4) Reipurth \& Gee 1986; (5) Ladd et al. 1991a; (6) Herbig \& Jones 1983; (7) Little et al. 1988; (8) Bally et al. 1995; (9) Gyulbudaghian et al. 1987; (10) Kun \& Prusti 1993; (11) Parker et al. 1991.

that the outflow from HH83 IRS has "blown out" of the molecular cloud and that it may be in a late stage of evolution. Interferometric CS observations (angular resolution $\sim 7^{\prime \prime}$ ) of the cloud core associated with HH83 IRS (Nakano et al. 1994) reveal a high-density structure consisting of a bar $\left(\sim 15^{\prime \prime}\right.$ long, centered on HH83 IRS and nearly perpendicular to the $\mathrm{HH}$ jet) and two ridges surrounding the base of the jet. These authors interpret the CS bar as a rotating circumstellar disk and the two ridges as tracing a small elongated hollow that may be playing an important role in focusing the $\mathrm{HH}$ jet.

We have detected a weak $\mathrm{NH}_{3}$ clump with the emission peak close to the proposed exciting source of $\mathrm{HH} 83$ (see Fig. 5). The $\mathrm{NH}_{3}$ clump appears unresolved with our beam of $\sim 1$ '.4, and we cannot study the small scale structure observed in CS by Nakano et al. (1994). At a larger scale, the weakest $\mathrm{NH}_{3}$ emission seems to extend further to the north and to the west, outside our mapped area. The region we mapped in $\mathrm{NH}_{3}$ corresponds to the core of the molecular clump studied in ${ }^{13} \mathrm{CO}$ by Bally et al. (1987, 1994), which also shows a clear northern extension.

\section{3. $\mathrm{HH} 84$}

This HH object was identified and studied by Reipurth $(1985,1989)$. HH 84 is a long $\left(\sim 101^{\prime \prime}\right)$ chain of HH knots outlining a well-collimated optical flow with a position angle of $\sim 154^{\circ}$. Unlike the large majority of the optical jets, this $\mathrm{HH}$ outflow is redshifted with respect to the ambient cloud. Morgan et al. (1991) found evidence for red wing emission in $\mathrm{CO}$ profiles, but could not confirm whether this is a molecular outflow. Up to now, no good candidate for the HH flow excitation has been proposed. IRAS 05317-0638 (identified with SW Ori, an $\mathrm{H} \alpha$ star and X-ray source; Strom et al. 1989a) lies $\sim 2^{\prime}$ south along the jet axis (see Fig. 6). However, based on the morphology of the knots, Reipurth (1989) argues that the flow exciting source should most probably be an embedded source (still undetected) located to the north of the jet.

We found weak ammonia emission associated with the HH knots. Our $\mathrm{NH}_{3}$ map is presented in Fig. 6. The ammonia emission maximum is close to the northern edge of the jet, and may be tracing the location of a possible embedded exciting source, then supporting the suggestion of 


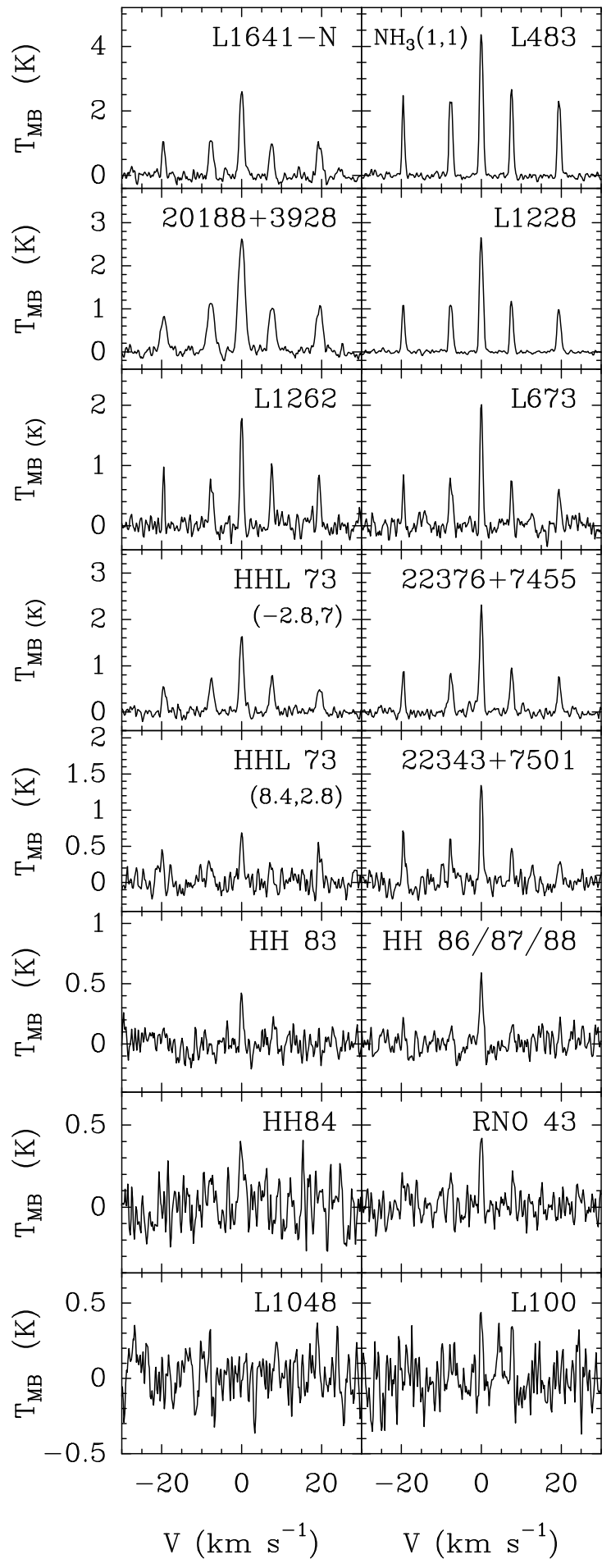

Fig. 1. Spectra of the $(J, K)=(1,1)$ inversion transition of the $\mathrm{NH}_{3}$ molecule towards the positions given in Table 2, for the detected sources. The vertical axis is the main beam brightness temperature and the horizontal axis is the velocity with respect to that of the center of the main line (given in Table 2)

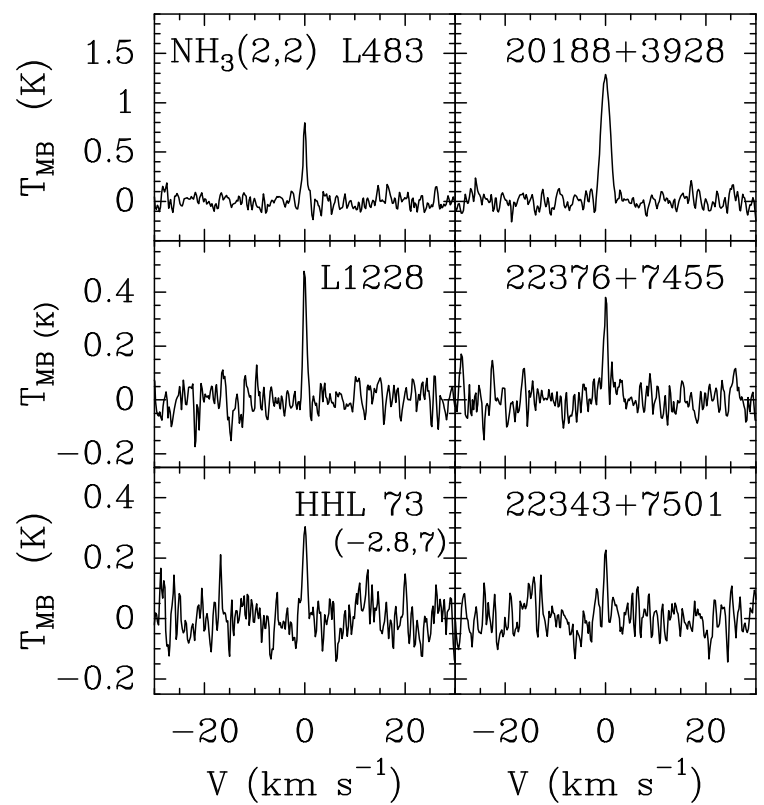

Fig. 2. Same as Fig. 1, for the $(J, K)=(2,2)$ inversion transition

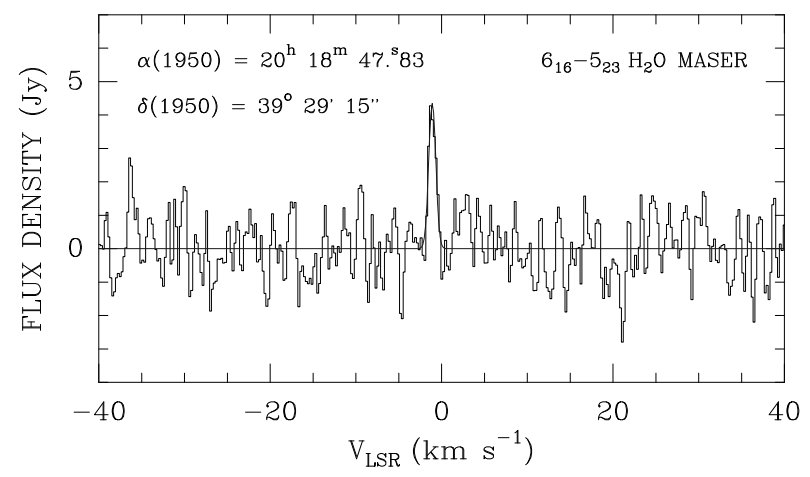

Fig. 3. Spectrum of the $\mathrm{H}_{2} \mathrm{O}$ maser detected near IRAS $20188+3928$, towards the position $\alpha(1950)=20^{\mathrm{h}} 18^{\mathrm{m}} 47^{\mathrm{s}} .83$, $\delta(1950)=39^{\circ} 29^{\prime} 15^{\prime \prime}$, as observed in Feb. 10, 1990

Reipurth (1989). However, we only mapped a small region and, in particular, our map does not reach the position of the IRAS source. The $\mathrm{NH}_{3}$ emission appears to extend further to the northeast, roughly following the distribution of the emission of the molecular cloud mapped in ${ }^{13} \mathrm{CO}$ by Bally et al. (1987; see detail in Reipurth 1989).

\section{4. $H H 86 / 87 / 88$}

The Herbig-Haro objects HH 86, HH 87 and HH 88 appear to be closely associated, and probably originate in the same flow (Reipurth 1985, 1989). The three objects are roughly aligned, but they exhibit a rather complex substructure and no local exciting source has been found. The knots in HH 86 appear to cluster around a faint star, but this star is probably not related to the flow, as 


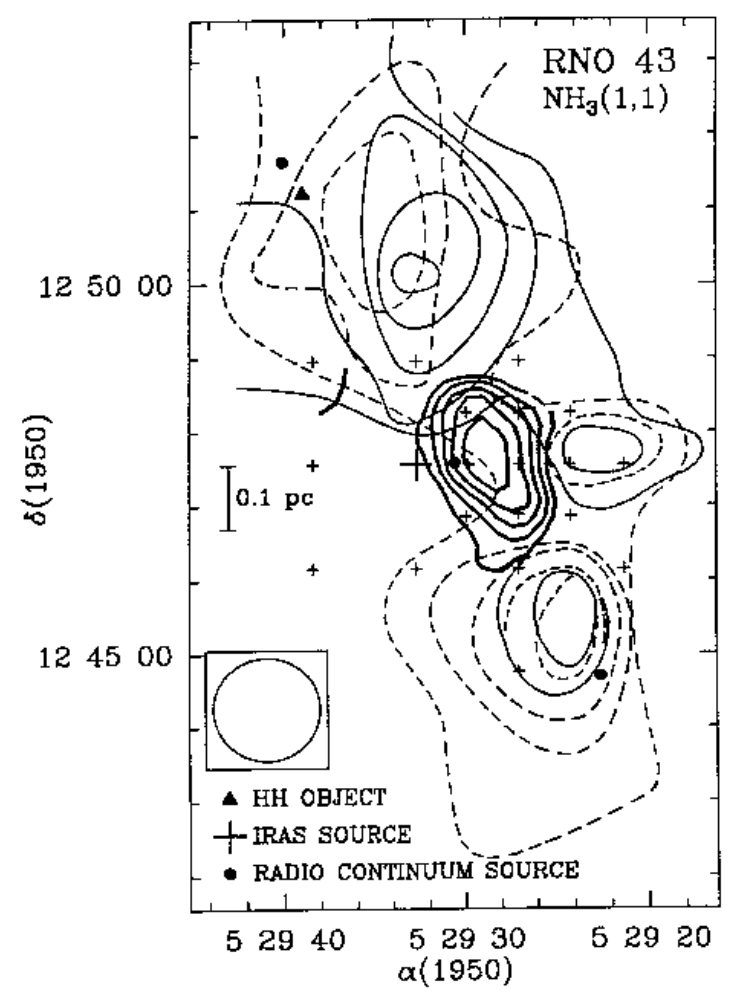

Fig. 4. Contour map of the peak antenna temperature of the main line of the ammonia $(J, K)=(1,1)$ inversion transition (thick line) in the RNO 43 region. The lowest contour level is $0.1 \mathrm{~K}$, and the increment is $0.02 \mathrm{~K}$. The observed positions are indicated with small crosses. The half power beam width of the telescope is shown as a circle. The positions of several relevant objects in the region are indicated. The central part of the $\mathrm{CO}$ bipolar outflow mapped by Cabrit et al. (1988) is shown with thin lines (solid contours indicate blueshifted gas, and dashed contours indicate redshifted gas)

discussed by Reipurth (1989). The T Tauri star V573 Ori (possibly associated with IRAS 05332-0637; Weintraub 1990) is also close by, but it does not lie on the line traced by the HH jet. Bally \& Devine (1994) suggest that these $\mathrm{HH}$ objects are the southern end of a 3 pc long "superjet" emanating from the exciting source of HH 34.

Our $\mathrm{NH}_{3}$ map is shown in Fig. 7. As it can be seen in the figure, the $\mathrm{NH}_{3}$ emission is faint and displaced to the east of the HH 86/87/88 complex. As the HH 86/87/88 complex lies along a ridge with increasing column density to the east (as mapped in ${ }^{13} \mathrm{CO}$ by Bally et al. 1987), it is plausible that the $\mathrm{NH}_{3}$ emission continues further to the east of the region we have mapped. Since no $\mathrm{NH}_{3}$ emission is seen towards the HH complex, this lack of high-density gas associated with the $\mathrm{HH}$ objects seems to exclude that their exciting source could be an undetected deeply embedded object in this region, then favoring a non local origin for the HH excitation, as suggested by Bally \& Devine (1994).

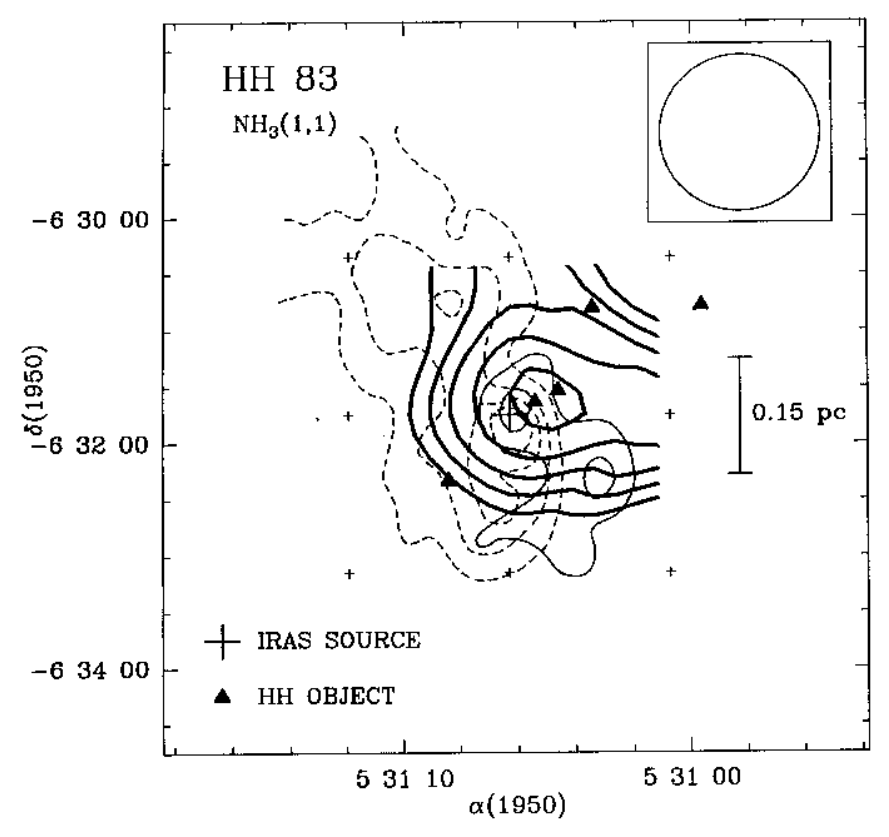

Fig. 5. Same as Fig. 4, for the $\mathrm{HH} 83$ region. The ammonia lowest contour level is $0.08 \mathrm{~K}$, and the increment is $0.02 \mathrm{~K}$. The map of the CO bipolar outflow is from Bally et al. (1994). The position of IRAS $05311-0631$ is indicated

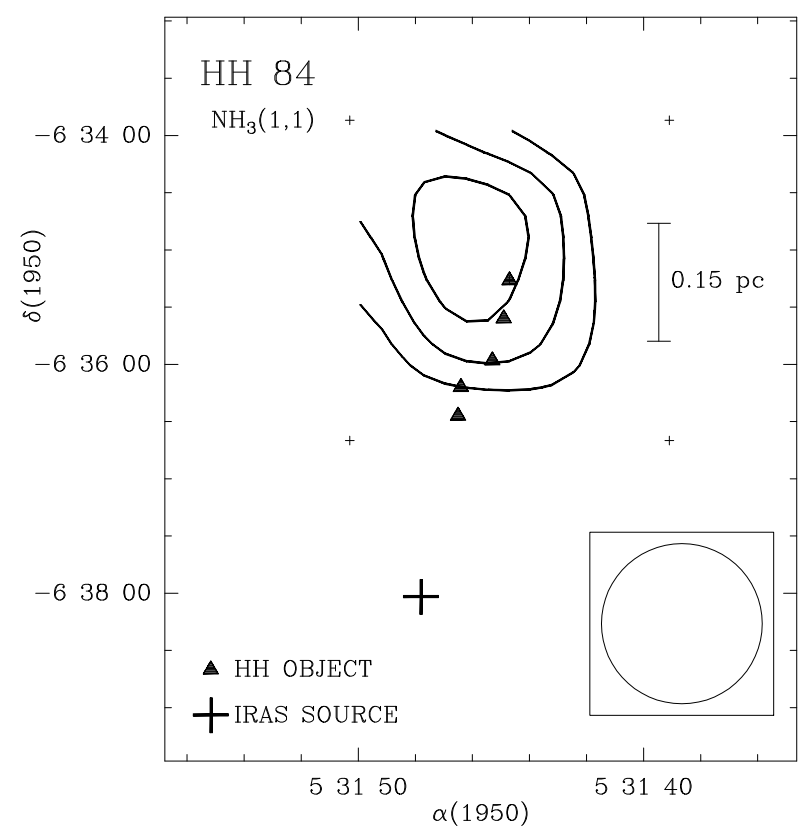

Fig. 6. Same as Fig. 4, for the HH 84 region. The ammonia lowest contour level is $0.08 \mathrm{~K}$, and the increment is $0.02 \mathrm{~K}$. The position of IRAS 05317 - 0638 (identified as SW Ori) is indicated 


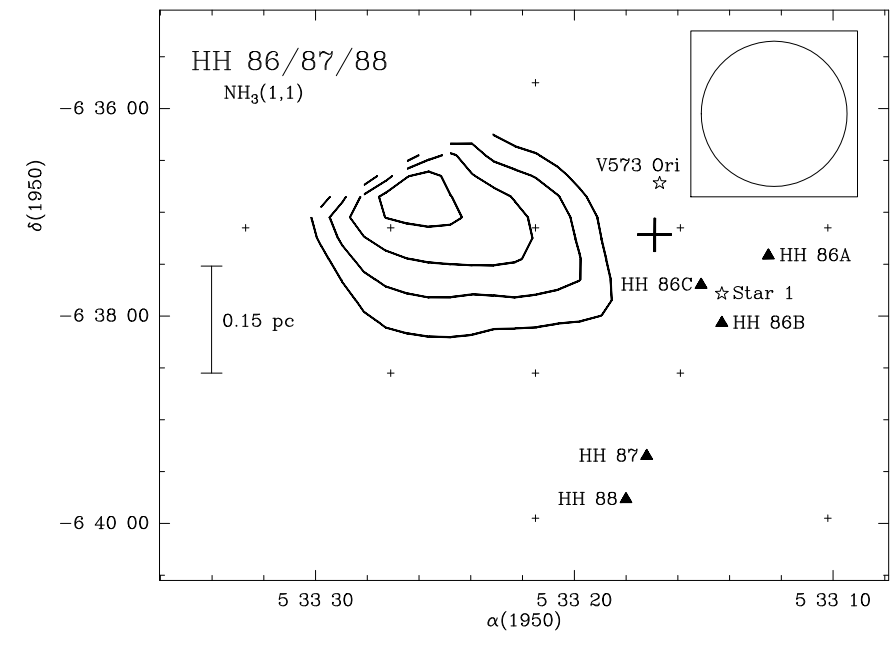

Fig. 7. Same as Fig. 4, for the HH 86/87/88 region. The ammonia lowest contour level is $0.12 \mathrm{~K}$, and the increment is 0.04 K. The position of IRAS $05332-0637$ (possibly associated with V573 Ori) is indicated

\section{5. $L 1641-N$}

A bipolar CO outflow, L1641-N (Fukui et al. 1986, 1988; Wilking et al. 1990), has been mapped in the northern part of the L1641 cloud. The outflow is centered on IRAS 05338-0624, which is proposed as the outflow exciting source. Although optically invisible, this source is one of the most luminous $\left(L_{\mathrm{IR}} \sim 220 L_{\odot}\right)$ IRAS sources in this region. Near-IR images (Strom et al. 1989c; Chen et al. 1993) reveal a dense stellar concentration centered on the IRAS position. The IRAS counterpart has been identified in the near-IR (Chen et al. 1993), millimeter (Wilking et al. 1989; Chen et al. 1995) and centimeter (Mundy et al. 1993; Anglada et al. 1996; Chen et al. 1995) ranges. Recently, $\mathrm{H}_{2} \mathrm{O}$ maser emission has been found in association with IRAS 05338 - 0624 (Xiang \& Turner 1995). In addition, several faint red nebulous objects of unknown nature have been found in a CCD image of the region (Reipurth 1985). A centimeter continuum source (Morgan et al. 1990; Anglada et al. 1996), also visible in the CCD image by Reipurth (1985), is found $\sim 2^{\prime}$ to the NE of the IRAS source, approximately midway between the red nebulous objects Re 35 and Re 43.

About $3^{\prime}$ to the SW of the center of the L1641-N molecular outflow, lies the source IRAS 05339-0626. This source $\left(L_{\text {bol }} \sim 86 L_{\odot}\right)$ is a typical Class I source (Strom et al. 1989a). Several near-IR sources (Chen et al. 1993) and a centimeter continuum source (Morgan et al. 1990 Anglada et al. 1996) are found near the IRAS position. Red wing $\mathrm{CO}$ emission has been observed towards this position, although it is unclear whether this high-velocity emission originates from IRAS $05339-0626$ or it is just extended high-velocity emission from the L1641-N outflow (Morgan et al. 1991).
We have mapped in $\mathrm{NH}_{3}$ a region that includes both IRAS sources. The map we have obtained is shown in Fig. 8. The $\mathrm{NH}_{3}$ structure consists of two subcondensations, peaking near the positions of the IRAS sources. This result suggests that both IRAS sources are embedded in dense gas. The condensation around IRAS 05338-0624 has been mapped in several molecular species (Fukui et al. 1988; Chen et al. 1992; Harju et al. 1991; McMullin et al. 1994) with the emission peaking close to the IRAS position as in our $\mathrm{NH}_{3}$ map. In particular, the $\mathrm{NH}_{3}$ map obtained by Harju et al. (1991) with higher angular resolution $\left(40^{\prime \prime}\right)$, is in good agreement with our results. The whole region encompassing both IRAS sources was mapped previously only in HCN (Takaba et al. 1986) and with lower $\left(\sim 3^{\prime}\right)$ angular resolution. CS emission was detected but not mapped, towards the two IRAS sources (Morgan \& Bally 1991).

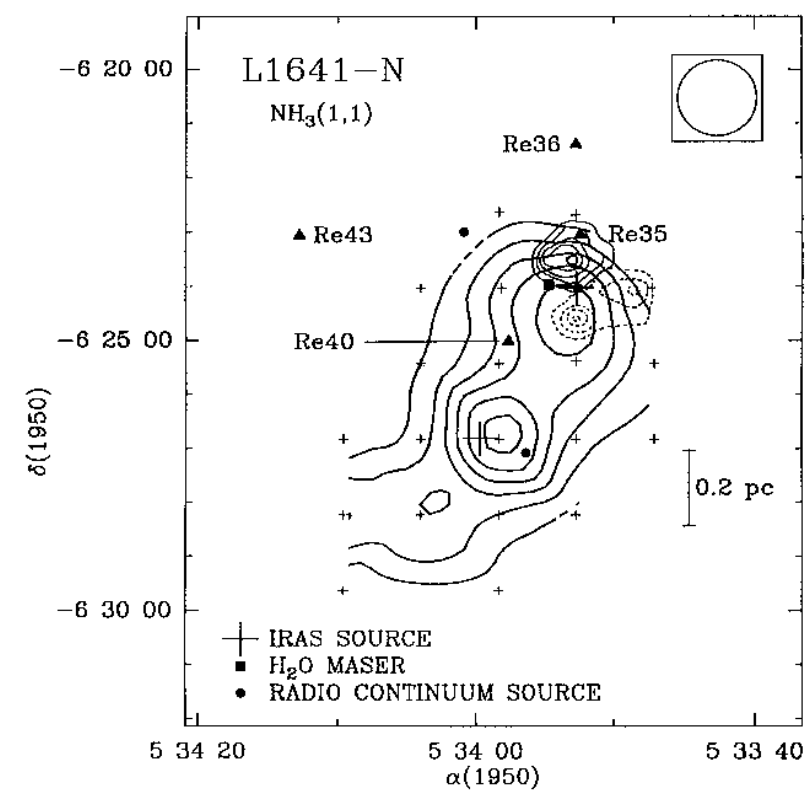

Fig. 8. Same as Fig. 4, for the L1641-N region. The ammonia lowest contour level is $0.15 \mathrm{~K}$, and the increment is $0.15 \mathrm{~K}$. The IRAS source associated with the northern ammonia peak is IRAS $05338-0624$ and the one associated with the southern ammonia peak is IRAS $05339-0626$. The map of the CO outflow is from Fukui et al. (1986)

\section{6. $L 100$}

L100 (Barnard 62) is a large, very opaque Bok globule in Ophiuchus, surrounded by bright rims. Reipurth \& Gee (1986), based on a photometric study, estimate a distance of $225 \pm 25 \mathrm{pc}$. These authors found several $\mathrm{H} \alpha$ emission stars associated with L100 and conclude that IRAS $17130-2053\left(0.25 L_{\odot} \leq L_{12-100} \leq 0.69 L_{\odot}\right)$, an IRAS source with no optical counterpart, represents the 
envelope of an embedded PMS object, more evolved than a protostar. Parker et al. (1988) detected a bipolar molecular outflow elongated in the NE-SW direction and centered at the position of IRAS $17130-2053$, which was proposed as the outflow exciting source.

We have mapped in $\mathrm{NH}_{3}$ the region around IRAS $17130-2053$. We have detected faint and unresolved $\mathrm{NH}_{3}(1,1)$ emission peaking near the position of the IRAS source. Our map is shown in Fig. 9. The IRAS position is near the maximum of the ammonia map, in agreement with the idea that it is an object embedded in high-density gas. However, the weakness of the ammonia emission suggests that it is associated with only a small amount of high-density gas and that the size of the ammonia clump could be much smaller than our beam.

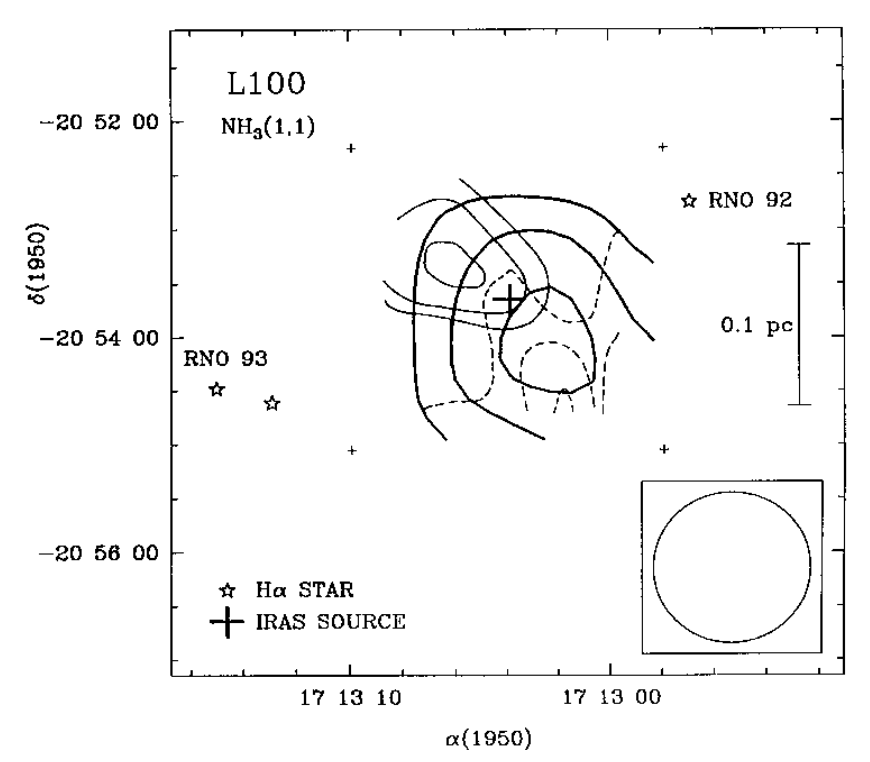

Fig. 9. Same as Fig. 4, for the L100 region. The ammonia lowest contour level is $0.08 \mathrm{~K}$, and the increment is $0.02 \mathrm{~K}$. The map of the CO outflow is from Parker et al. (1988)

\section{7. $L 483$}

Parker et al. $(1988,1991)$ and Fuller et al. (1995) have mapped in $\mathrm{CO}$ a compact bipolar molecular outflow in the L483 dark cloud. The outflow is clearly elongated along the $\mathrm{E}-\mathrm{W}$ direction and it is centered on the low-luminosity infrared source IRAS $18148-0440\left(L=14 L_{\odot}\right.$, assuming a distance of $200 \mathrm{pc}$; Ladd et al. 1991a), which is proposed as the outflow exciting source. This source has neither optical nor near-infrared counterpart (Parker 1991). The source has been detected with the VLA at $3.6 \mathrm{~cm}$ (Anglada et al. 1996). From the far-IR data and from submillimeter observations (Ladd et al. 1991b; Fuller et al. 1995), Fuller et al. (1995) conclude that the IRAS source is a very young object similar to the so-called Class 0 sources. The source is surrounded by a bipolar near-IR nebula, and a jet-like region of $\mathrm{H}_{2}$ emission, ending in a bright knot, extends along the blue lobe of the CO outflow (Fuller et al. 1995). An $\mathrm{H}_{2} \mathrm{O}$ maser near the IRAS position has been detected by Xiang \& Turner (1995) through single-dish observations.

In Fig. 10 we show the ammonia map obtained from our observations. The $\mathrm{NH}_{3}$ emission peaks very close to the IRAS source position. This fact, together with the infrared results (Fuller et al. 1995), suggest that the IRAS source is deeply embedded in the dense core, giving support to its identification as the powering source of the molecular outflow. Our ammonia map is in good agreement with the ammonia map shown by Fuller \& Myers (1993). Fuller \& Myers (1993) found two velocity components separated by $0.28 \mathrm{~km} \mathrm{~s}^{-1}$ in an $\mathrm{NH}_{3}$ spectrum obtained towards the peak of the core, and discuss on their relationship with the overall distribution of dense gas. The velocity resolution of our observations $\left(\sim 0.2 \mathrm{~km} \mathrm{~s}^{-1}\right)$ does not allow us to separate these components in the spectra. Goodman et al. (1993) report a velocity gradient $\left(\sim 1.9 \pm 0.2 \mathrm{~km} \mathrm{~s}^{-1} \mathrm{pc}^{-1}, \mathrm{PA}=52^{\circ}\right)$ in the region. From our data, we estimate a velocity gradient of $2-3 \mathrm{~km} \mathrm{~s}^{-1} \mathrm{pc}^{-1}$ approximately in the SW-NE direction, which is consistent with the result of Goodman et al. (1993).

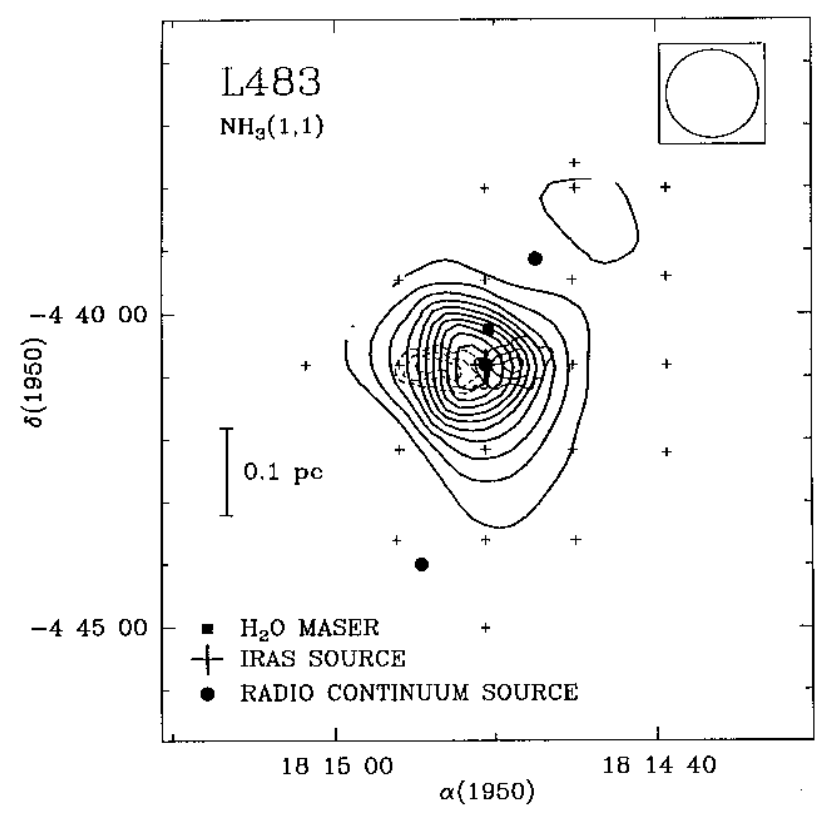

Fig. 10. Same as Fig. 4, for the L483 region. The ammonia lowest contour level is $0.20 \mathrm{~K}$, and the increment is $0.15 \mathrm{~K}$. The map of the CO outflow is from Parker et al. (1988) 


\section{8. $L 673$}

The distance to the L673 dark cloud is not well established. Estimates by different authors range between $150 \mathrm{pc}$ and $400 \mathrm{pc}$. We will adopt a distance of $300 \mathrm{pc}$, based on proper motions studies (Herbig \& Jones 1983). Armstrong \& Winnewisser (1989) discovered a very extended $\left(10^{\prime} \times 15^{\prime}\right)$ bipolar molecular outflow in the northern part of L673. There are four IRAS point sources within five arcmin of the center of the outflow. From an analysis of the IRAS colors, Armstrong \& Winnewisser conclude that IRAS $19184+1118$ is probably a visible mainsequence star, while the IRAS colors of the other three sources (IRAS $19180+1116,19180+1114,19181+1112$ ) are consistent with those of embedded stars. In particular, the source IRAS $19180+1116$, which coincides with RNO 109 (Cohen 1980), is proposed by Armstrong \& Winnewisser (1989) as the most likely candidate to be the outflow exciting source. Ladd et al. (1991a, b) obtained farinfrared photometry and images of IRAS $19180+1116$ and IRAS $19180+1114$, showing that a large fraction of the luminosity of these objects is radiated at long wavelengths $(\lambda>60 \mu \mathrm{m})$, indicating that they are very young.

Our ammonia map is shown in Fig. 11. The ammonia structure consists of three subcondensations, peaking near the position of the sources IRAS $19180+1116$ (RNO 109), IRAS $19180+1114$ and IRAS $19181+1112$. This result suggests that the three sources are embedded in dense gas. The source IRAS $19180+1114$ is located very close to the strongest emission maximum, suggesting that this source is associated with the largest amount of highdensity gas, being probably the most deeply embedded of these three objects. The spectral energy distribution of this source (Armstrong \& Winnewisser 1989; Ladd et al. 1991a) is also consistent with this suggestion. The source IRAS $19180+1114$ is also well centered in between the two outflow lobes, despite of the irregular geometry of the molecular outflow (see Fig. 11). Taking into account the ammonia results, the spectral energy distribution, and the location with respect to the CO outflow, we favor IRAS $19180+1114$ (rather than IRAS $19180+1116=$ RNO 109) as the best candidate for the excitation of the molecular outflow in the region.

Although our observations do not reach the position of IRAS $19184+1118$, from the region we have mapped it seems clear that the $\mathrm{NH}_{3}$ emission decreases as one moves to the NE, towards the position of this IRAS source. Thus, this source does not appear to be associated with a significant amount of dense molecular gas. This result is confirmed by the CS $(J=1 \rightarrow 0)$ observations of Morata et al. (1996). Even though the CS emission appears to be more extended than the $\mathrm{NH}_{3}$ emission, the CS map shows that IRAS $19184+1118$ lies at the outer edge of the CS distribution, suggesting that this source is not embedded in high density gas. These results are in agreement with those of Armstrong \& Winnewisser (1989), which concluded that the IRAS colors of this source are typical of a visible main-sequence star.

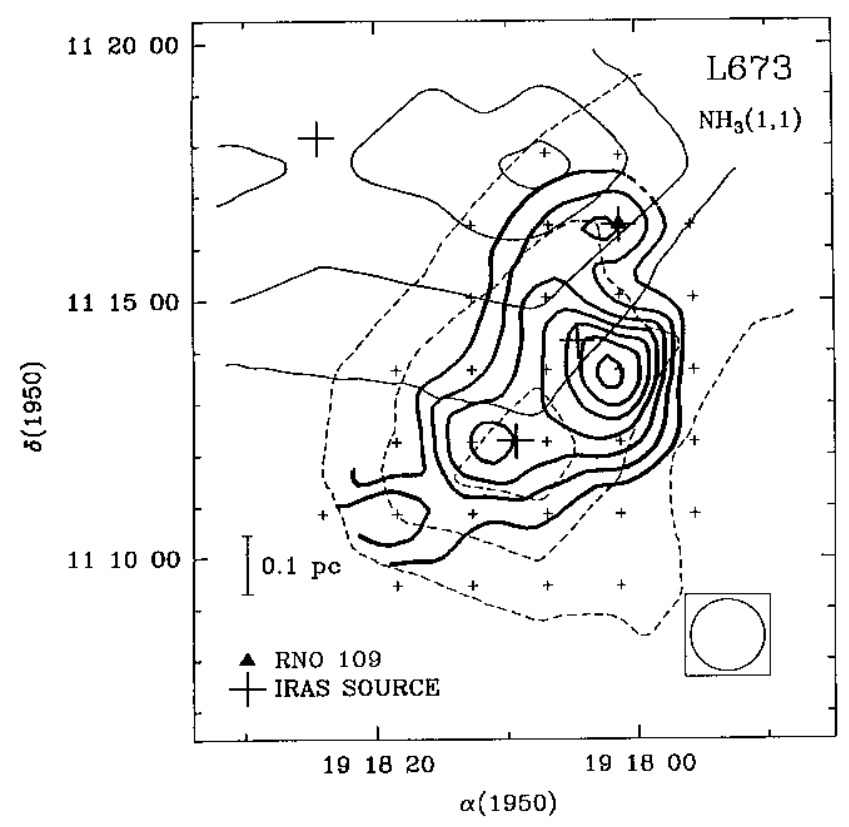

Fig. 11. Same as Fig. 4, for the L673 region. The ammonia lowest contour level is $0.15 \mathrm{~K}$, and the increment is $0.1 \mathrm{~K}$. The IRAS sources associated with the ammonia structure are (from north to south) IRAS $19180+1116$ (RNO 109), IRAS $19180+1114$ and IRAS $19181+1112$. The IRAS source outside the ammonia structure is IRAS $19184+1118$. The map of the CO outflow is from Armstrong \& Winnewisser (1989)

\subsection{IRAS 20188+3928}

IRAS $20188+3928\left(L=510^{4}(D / 4 \mathrm{kpc})^{2} L_{\odot}\right.$; Odenwald \& Schwartz 1993) is associated with a compact molecular cloud located in the Cygnus region. The distance to this source is very uncertain $(0.4 \mathrm{kpc} \leq D \leq 4 \mathrm{kpc}$; Little et al. 1988). We adopt in this paper its largest value $(4 \mathrm{kpc})$; thus, most of the physical parameters obtained for this region will be upper limits. Little et al. (1988) mapped in ${ }^{12} \mathrm{CO}$ and $\mathrm{HCO}^{+}$a bipolar molecular outflow associated with this IRAS source, concluding that the outflowing gas has a dense and clumpy nature.

We have detected a compact ammonia condensation (Fig. 12), with the emission peaking very close to the IRAS $20188+3928$ position. This result suggests that the IRAS source is deeply embedded in the high density gas, as usually are the exciting sources of molecular outflows. The ammonia lines in this condensation are significantly wider $\left(\Delta V \sim 1.5-2 \mathrm{~km} \mathrm{~s}^{-1}\right)$ than in the other regions of our sample (see Table 2). Moreover, there is a northsouth velocity gradient in the $\mathrm{NH}_{3}$ condensation, with the line velocity in the northern part being redshifted (by 
$\sim 0.5 \mathrm{~km} \mathrm{~s}^{-1}$ ) with respect to the southern part, i.e., roughly in the same direction as the outflow. These results suggest that the dense gas around IRAS $20188+3928$ is perturbed by the outflow from this star and is entrained into the high velocity gas, in agreement with the $\mathrm{HCO}^{+}$ results obtained by Little et al. (1988).

Palla et al. (1991) detected $\mathrm{H}_{2} \mathrm{O}$ maser emission on January 30, 1989 in a single observation towards the position of the IRAS source, obtaining a peak line flux $S_{\nu} \simeq 45$ Jy and a radial velocity $V_{\mathrm{LSR}}=-1.1 \mathrm{~km} \mathrm{~s}^{-1}$. We detected this $\mathrm{H}_{2} \mathrm{O}$ maser on February 10, 1990. From a seven-point map centered on the IRAS position, we found that the maximum of emission was displaced $\sim 1^{\prime}$ to the $\mathrm{NW}$ of the IRAS source. In Fig. 3, we show the spectrum of the $\mathrm{H}_{2} \mathrm{O}$ maser towards this position. The single feature we observed can be fitted with a Gaussian profile having peak line flux $S_{\nu}=4.4 \pm 0.9 \mathrm{Jy}$, half-power full width $\Delta V=1.0 \pm 0.1 \mathrm{~km} \mathrm{~s}^{-1}$, and radial velocity with respect to the local standard of rest, $V_{\mathrm{LSR}}=-1.09 \pm 0.07 \mathrm{~km} \mathrm{~s}^{-1}$. Thus, this $\mathrm{H}_{2} \mathrm{O}$ maser feature shows high variability on a time scale of one year. Several new maser features, at different velocities appeared also in observations carried out in 1990 and 1991 (Xiang \& Turner 1995). One of these features coincides within $4^{\prime \prime}$ with the position of the IRAS source.

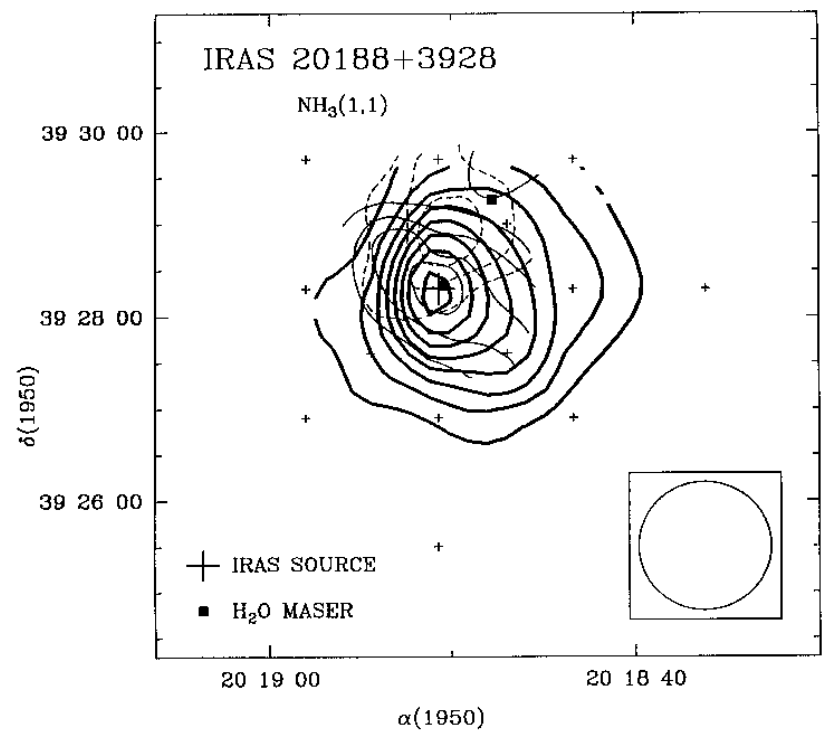

Fig. 12. Same as Fig. 4, for the IRAS $20188+3928$ region. The ammonia lowest contour level is $0.20 \mathrm{~K}$, and the increment is $0.1 \mathrm{~K}$. The map of the CO outflow is from Little et al. (1988)

\subsection{0. $L 1228$}

L1228 is a high galactic latitude dark cloud, whose distance is poorly known. Haikala et al. (1991) estimate a distance between 100 and 200 pc, while Bally et al. (1995) argue that a better value is $300 \mathrm{pc}$. In this paper we have adopted this last value. Haikala \& Laureijs (1989) discovered a large $\left(18^{\prime} \times 9^{\prime} \simeq 1.6 \mathrm{pc} \times 0.8 \mathrm{pc}\right.$, for the assumed distance of $300 \mathrm{pc}$ ) and well-collimated bipolar $\mathrm{CO}$ outflow, with its axis in the NE-SW direction. The outflow is centered on the low luminosity object IRAS $20582+7724$ $\left(L \sim 4 L_{\odot}\right)$, which was proposed as the outflow exciting source. Anglada et al. (1996) have detected this source in the radio continuum at $3.6 \mathrm{~cm}$ with the VLA. Very recently, Bally et al. (1995) have obtained a new CO map of the outflow and detected several $\mathrm{HH}$ objects along the axis of the molecular outflow. Moreover, these authors detected an $\mathrm{H}_{2}$ jet emerging from IRAS $20582+7724$, but the jet axis differs from that of the molecular outflow by $\sim 40^{\circ}$, suggesting that the jet ejection direction varies over time. Bally et al. (1995) also detected a long highly collimated $\mathrm{HH}$ jet (HH 200), which may be associated with a very low velocity and faint blueshifted CO lobe, and whose exciting source appears to be an embedded T Tauri star located $\sim 1.5 \mathrm{NW}$ of the IRAS source.

Tafalla et al. (1994) have observed the dense gas around the IRAS source as traced by $\mathrm{C}_{3} \mathrm{H}_{2}$ and $\mathrm{HCN}$. These authors found sudden shifts in the line velocity of these molecules, with a systematic velocity pattern that agrees in direction and velocity sense with the $\mathrm{CO}$ outflow. Tafalla et al. identified three distinct velocity components in the core, and interpreted these results as evidence for the disruption of the dense core by the bipolar outflow from the IRAS source.

Our ammonia map of this region (Fig. 13) shows a condensation of $\sim 3^{\prime} \times 2^{\prime}$ in size, elongated in the N-S direction. The ammonia emission peaks very close to the position of the IRAS and radio continuum source, suggesting that this source is deeply embedded in the high density gas, as it is expected for the exciting source of the outflow. For positions with good signal-to-noise ratio, our data show a velocity shift in the ammonia line velocity, consistent with the direction and sense of the bipolar molecular outflow. In Fig. 14 we show the spectra of the $\mathrm{NH}_{3}(1,1)$ main line observed towards the position of the IRAS source, as well as towards two additional positions displaced 1.'4 to the east and 1.'4 to the west, respectively. As it can be seen in the figure, the central velocity of the lines are clearly displaced, with a velocity shift between the extreme positions of $\sim 0.54 \mathrm{~km} \mathrm{~s}^{-1}$, corresponding to a velocity gradient of $\sim 2.2 \mathrm{~km} \mathrm{~s}^{-1} \mathrm{pc}^{-1}$. Tafalla et al. (1994), from their higher angular resolution data, obtained velocity gradients of up to $10 \mathrm{~km} \mathrm{~s}^{-1} \mathrm{pc}^{-1}$ on a scale of $20^{\prime \prime}$. Our lower angular resolution data do not allow us to detect these sudden shifts in velocity but, in any case, they provide evidence that the dense gas is perturbed and accelerated by the molecular outflow. 


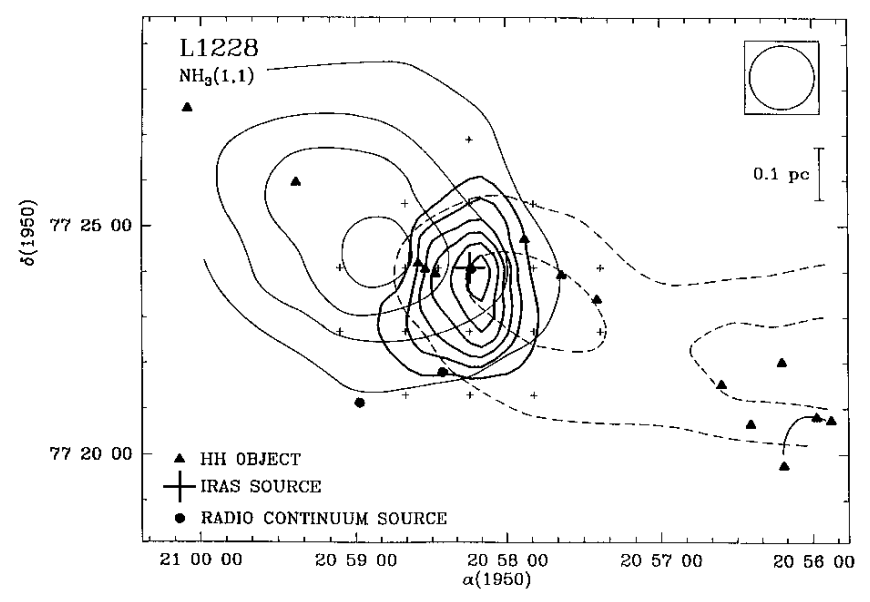

Fig. 13. Same as Fig. 4, for the L1228 region. The ammonia lowest contour level is $0.20 \mathrm{~K}$, and the increment is $0.15 \mathrm{~K}$. The map of the CO bipolar outflow is from Haikala \& Laureijs (1989)

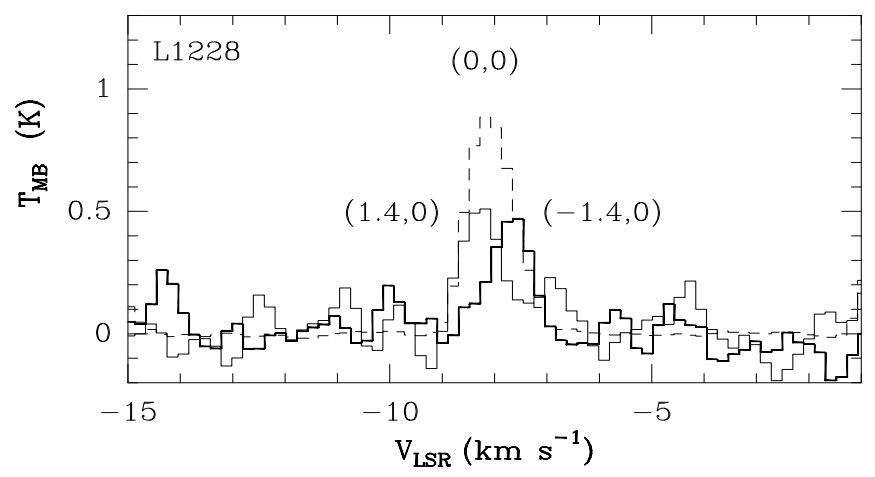

Fig. 14. Spectra of the $\mathrm{NH}_{3}(1,1)$ main line towards three selected positions in L1228. The offsets are in arc minutes with respect to the IRAS position. To make easiest the comparison, the main beam brightness temperature of the $(0,0)$ spectrum has been divided by 3

\subsection{1. $H H L 73$}

HHL 73 is an Herbig-Haro like object, whose position coincides, within observational errors, with an $\mathrm{H}_{2} \mathrm{O}$ maser (Gyulbudaghian et al. 1987) and with the source IRAS $21432+4719$. A region of $\sim 9^{\prime} \times 7^{\prime}$ around HHL 73 was mapped in ammonia by Verdes-Montenegro et al. (1989), using also the Haystack radio telescope. Their observations revealed a condensation with an angular size of $\sim 4.7 \times 2$ ' 2 , elongated in the NW-SE direction, and with the HHL 73 object located very close to the ammonia emission peak. Verdes-Montenegro et al. (1989) detected also a weaker ammonia condensation, located $\sim 5^{\prime}$ northeast of the main condensation. No signs of star formation associated with this second clump are known at present.

The region was mapped in CS by Pastor et al. (1991). The CS emitting region is elongated in the E-W direction and it is more extended $\left(23^{\prime} \times 7^{\prime}\right)$ than the region mapped in ammonia by Verdes-Montenegro et al. (1989). The CS structure presents several emission peaks, three of them coinciding with IRAS $21429+4726$, IRAS $21432+4719$ and IRAS $21441+4722$. These IRAS sources have faint optical counterparts on the Palomar Sky Survey red print and were classified as "protostar type" by Dobashi et al. 1992. Recently, Dobashi et al. (1993) have detected three highly asymmetric molecular outflows associated with these IRAS sources.

To complete the study in $\mathrm{NH}_{3}$ of this region, we carried out new observations, completing those of VerdesMontenegro et al. (1989), in order to cover the overall region observed in $\mathrm{CS}$. In Fig. 15 we show the complete $\mathrm{NH}_{3}$ map of the region (including the data of VerdesMontenegro et al. 1989). Four ammonia clumps, coinciding with emission peaks in the CS map of Pastor et al. (1991), are observed in the figure. Three of the ammonia clumps are associated with an IRAS source, located very close to the emission peak, thus suggesting that these clumps contain a young embedded object. The mean separation between these clumps $(\sim 1-2 \mathrm{pc})$ is of the order of the typical distance between stars. Therefore, this region appears to be an example of simultaneous formation of several stars in the same cloud, as it was suggested by Pastor et al. (1991).

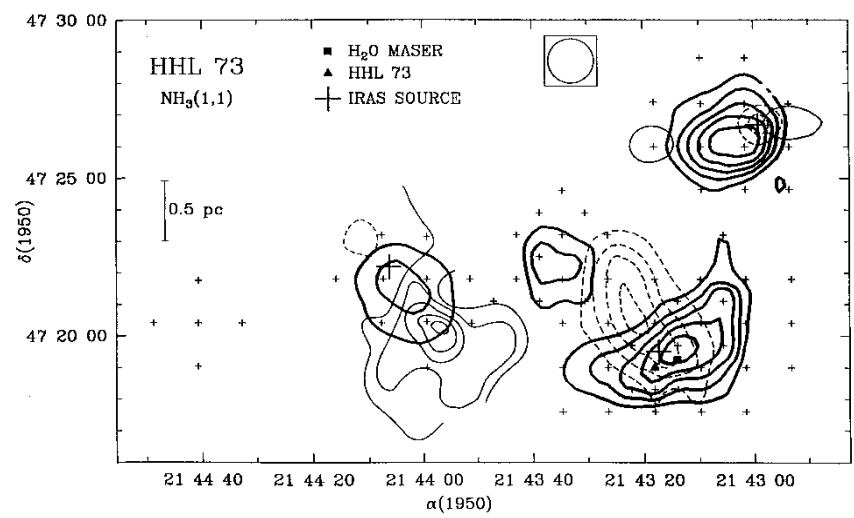

Fig. 15. Same as Fig. 4, for the HHL 73 region. The ammonia lowest contour level is $0.12 \mathrm{~K}$, and the increment is $0.1 \mathrm{~K}$. The $\mathrm{NH}_{3}$ map obtained by Verdes-Montenegro et al. (1989) is also included. The maps of the CO outflows are from Dobashi et al. (1993)

\subsection{2. $L 1251$}

L1251 is an elongated dark cloud (Lynds 1962) apparently belonging to the "Cepheus Flare" giant molecular cloud complex (Lebrun 1986). The estimated distance of this cloud is between $200 \mathrm{pc}$ to $500 \mathrm{pc}$. We have adopted a distance of 300 pc, estimated from a photometric study by Kun \& Prusti (1993). Several indications of low-mass star formation have been found in L1251, with several $\mathrm{H} \alpha$ 


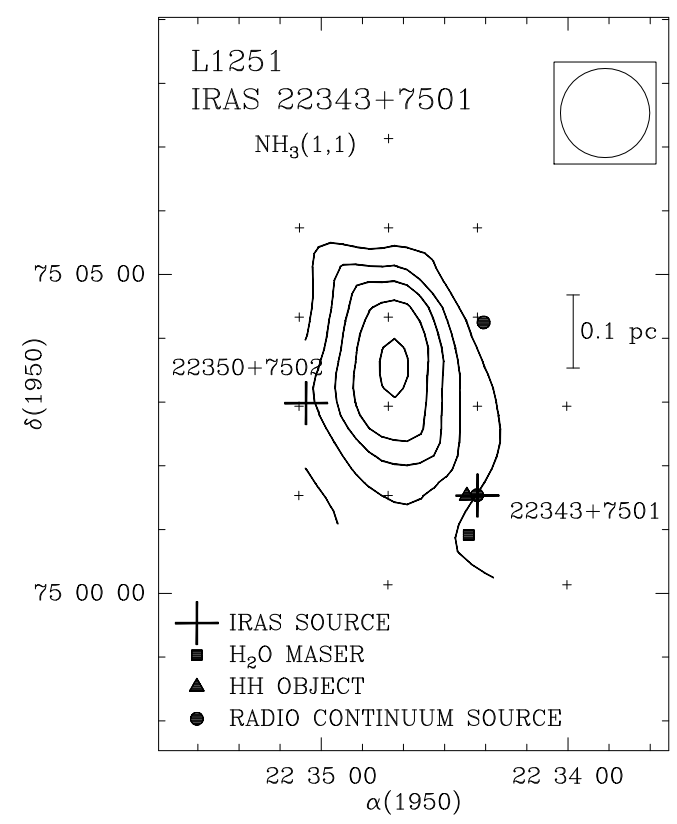

Fig. 16. Same as Fig. 4, for the IRAS $22343+7501$ region in L1251. The ammonia lowest contour level is $0.1 \mathrm{~K}$, and the increment is $0.1 \mathrm{~K}$

emission stars and infrared point sources detected in the cloud (see Kun \& Prusti 1993 and references therein). Our following study of this region focuses on two IRAS sources, IRAS $22343+7501$ and IRAS $22376+7455$, which are the most luminous sources in L1251 and appear to be the powering sources of bipolar CO outflows.

\subsubsection{IRAS $22343+7501$}

IRAS $22343+7501$ is the driving source of an extended and poorly collimated outflow, L1251-A (Schwartz et al. 1988; Sato \& Fukui 1989). The outflow map differs from one study to the other. The outflow map obtained by Sato \& Fukui (1989) represents molecular gas with relatively low velocity $\left(\lesssim 4 \mathrm{~km} \mathrm{~s}^{-1}\right)$ and is extended $(\sim 1.1 \mathrm{pc})$, with the axis approximately in the NE-SW direction. On the other hand, the map obtained by Schwartz et al. (1988), with higher angular resolution, includes higher velocity gas $\left(\lesssim 8 \mathrm{~km} \mathrm{~s}^{-1}\right)$, is more compact and shows a clear asymmetry in the intensity the two lobes.

Balázs et al. (1992) detected several Herbig-Haro objects (apparently forming an optical jet with its axis coincident with that of the $\mathrm{CO}$ outflow), and propose that the exciting source of these objects is also IRAS $22343+7501$. Anglada et al. (1996) have detected this source in the radio continuum at $3.6 \mathrm{~cm}$. Xiang \& Turner (1995) have detected an $\mathrm{H}_{2} \mathrm{O}$ maser within $\sim 0$ '. 6 of the IRAS position.

The source IRAS $22350+7502$ (probably a T Tauri star; Kun \& Prusti 1993) lies about $2^{\prime}$ to the north-east of IRAS $22343+7501$.

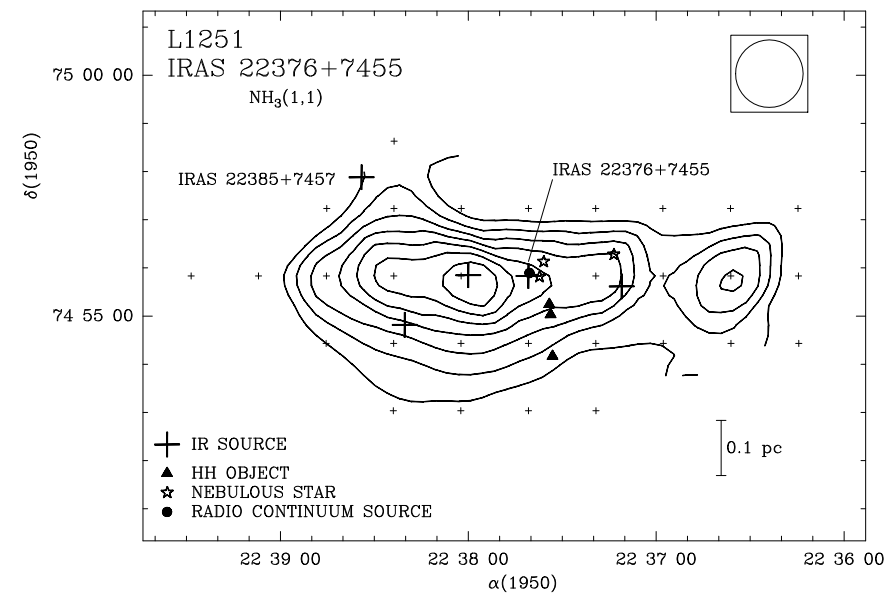

Fig. 17. Same as Fig. 4, for the IRAS $22376+7455$ region in L1251. The lowest contour level is $0.15 \mathrm{~K}$, and the increment is $0.1 \mathrm{~K}$

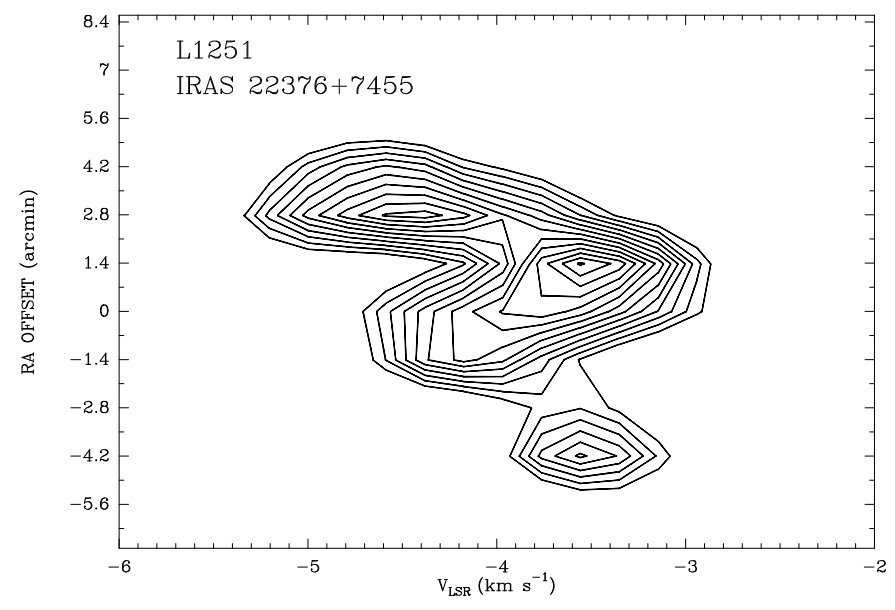

Fig. 18. Position-velocity diagram of the $\mathrm{NH}_{3}(1,1)$ main line along the major axis $\left(\mathrm{PA}=0^{\circ}\right)$ of the condensation associated with IRAS $22376+7455$, in L1251. The lowest contour level is $0.2 \mathrm{~K}$ and the increment is $0.05 \mathrm{~K}$. Right ascension offsets are from the position of IRAS $22376+7455$

In Fig. 16, we show our ammonia map. The two IRAS sources lie at the edge of the $\mathrm{NH}_{3}$ condensation, being displaced by $\sim 2^{\prime}(\sim 0.1 \mathrm{pc})$ from the emission peak. At present, no source coincident with the ammonia emission peak has been found. As the emission peak is displaced from the outflow center, it is not likely that the outflow exciting source coincides with this ammonia maximum. The fact that the source proposed as the exciting source of the outflow, IRAS $22343+7501$, is displaced $\sim 0.1 \mathrm{pc}$ in projection from the ammonia emission peak may be due either because the outflow has disrupted part of the cloud core, or because the source was formed in the dense cloud but has escaped out of the clump (for a velocity of the star with respect to the cloud of $\sim 1 \mathrm{~km} \mathrm{~s}^{-1}$, a time of $\sim 10^{5} \mathrm{yr}$, which is similar to the time-scale of the outflow, 
is required to cover the observed displacement). We note that Morata et al. (1996) have mapped this region in CS, obtaining that the CS extends over a region larger than the $\mathrm{NH}_{3}$ with IRAS $22343+7501$ near a CS emission peak.

\subsubsection{IRAS $22376+7455$}

$\mathrm{CO}$ observations in the region associated with this source (Sato \& Fukui 1989; Sato et al. 1994) have revealed a well collimated and very compact bipolar outflow, L1251-B, extending over $4^{\prime}$ in the NW-SE direction. IRAS $22376+$ $7455\left(L_{\mathrm{FIR}}=14 L_{\odot}\right.$; Sato et al. 1994) lies near the center of the two lobes, suggesting that is driving the bipolar outflow. IRAS $22376+7455$, apparently without optical counterpart (Kun \& Prusti 1993; Eiroa et al. 1994b), is detected in the radio continuum at $3.6 \mathrm{~cm}$ by Anglada et al. (1996), and is likely to be a protostar embedded in a dense molecular cloud core (Sato et al. 1994). Eiroa et al. (1994b) found several Herbig-Haro objects (HH 189A, B and C) that may be associated with IRAS $22376+7455$ or with a nearby nebulous star. Kun \& Prusti (1993) and Eiroa et al. (1994b) also found several infrared sources and $\mathrm{H} \alpha$ emission stars in this region.

In Fig. 17 we show our ammonia map. The observed $\mathrm{NH}_{3}$ condensation appears to be very elongated $\left(\sim 8^{\prime} \times 2^{\prime}\right)$ in the east-west direction. IRAS $22376+7455$ is located in the midst of the high density gas, confirming that it is an embedded source. However, this source is displaced $\sim 1^{\prime}$ $(\sim 0.1 \mathrm{pc})$ from the $\mathrm{NH}_{3}$ emission peak. Several other infrared sources are observed towards the $\mathrm{NH}_{3}$ condensation, suggesting that they represent embedded sources. One of these sources coincides positionally with the $\mathrm{NH}_{3}$ emission peak. There is a secondary emission peak (located $\sim 6^{\prime}$ to the west of the main one) that appears not to be associated with any known infrared source. The source IRAS $22385+7457$ is located $\sim 4^{\prime}$ to the northeast of IRAS $22376+7455$, at the edge of the $\mathrm{NH}_{3}$ structure, suggesting that it is not deeply embedded in the dense cloud. This source is associated positionally with an $\mathrm{H} \alpha$ emission star, and it is proposed to be a T Tauri star (Kun $\&$ Prusti 1993), in agreement with the suggestion that this source is more evolved than the sources still deeply embedded in dense gas.

From our $\mathrm{NH}_{3}$ data, we found a velocity gradient of $\sim 1.4 \mathrm{~km} \mathrm{~s}^{-1} \mathrm{pc}^{-1}$ in the NE-SW direction, with sudden velocity shifts of up to $1 \mathrm{~km} \mathrm{~s}^{-1}$ (corresponding to gradients of $8 \mathrm{~km} \mathrm{~s}^{-1} \mathrm{pc}^{-1}$ ) along the region. In Fig. 18 we show a position-velocity diagram along the major axis of the clump. The velocity gradient does not follows the $\mathrm{NH}_{3}$ condensation axis, but it has a complex bidimensional structure, so that we do not think that it is due to a global rotational motion of the dense condensation. Goodman et al. (1993) from $\mathrm{NH}_{3}$ observations, Morata et al. (1996) from CS observations, and Sato \& Fukui (1989) from $\mathrm{C}^{18} \mathrm{O}$, also found the existence of a velocity gradient in this region.
In Fig. 19, we show the overall L1251 region, enclosing the two ammonia condensations we have mapped and their associated molecular outflows. Note that these two condensations coincide with the two brightest spots in the $\mathrm{C}^{18} \mathrm{O}$ map by Sato et al. (1994).

\subsection{3. $L 1262$}

L1262 is an isolated Bok globule with a very high visual extinction (Lynds 1962) located at a distance of $200 \mathrm{pc}$ (Parker et al. 1991). Parker et al. (1988, 1991) discovered a well-collimated bipolar molecular outflow approximately 2.5 in extent along its axis. Interferometric ${ }^{12} \mathrm{CO}$ observations (Terebey et al. 1989) show a more compact $\left(\sim 20^{\prime \prime}\right)$ outflow. The outflow is elongated in the northeastsouthwest direction and is centered at the position of the source IRAS $23238+7401$, which is proposed as the exciting source of the outflow. This IRAS source has been classified as a Class I embedded source without optical counterpart (Parker 1991). The velocity of the outflow decreases gradually as one moves away from the source (Parker et al. 1988). Anglada et al. (1996) found two radio continuum sources at $3.6 \mathrm{~cm}$, one of them being associated with the IRAS source.

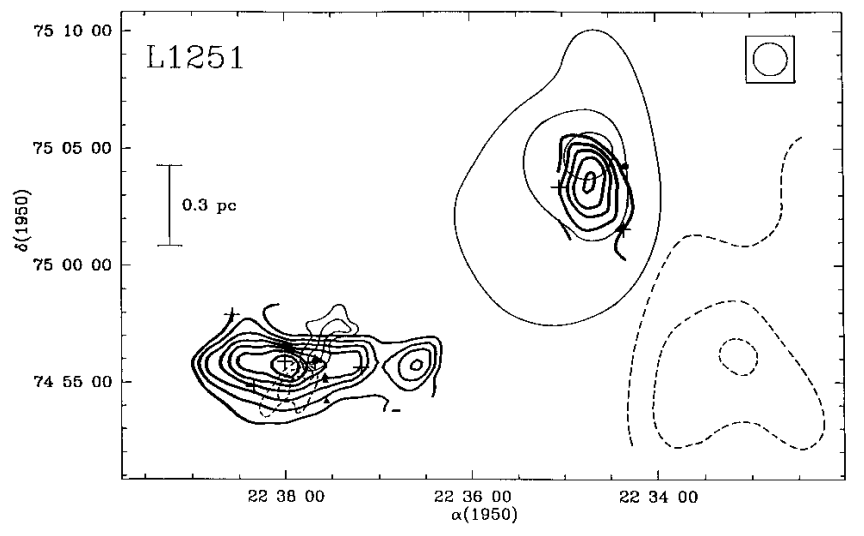

Fig. 19. Region enclosing the two ammonia dense cores observed in L1251 (Figs. 16 and 17). Superposed are shown the two associated CO outflows, L1251-A and L1251-B (Sato et al. 1994)

Our $\mathrm{NH}_{3}$ map is shown in Fig. 20. The $\mathrm{NH}_{3}$ condensation presents two emission peaks, one of them coinciding with the source IRAS $23238+7401$. Our map is similar to the map obtained by Benson et al. (1984) using the same ammonia inversion line, but our sensitivity is slightly better and we are able to distinguish two emission peaks. Zhou et al. (1989) observed this region in CS, $J=2 \rightarrow 1$ and $J=3 \rightarrow 2$, obtaining that the CS emission is more extended than the $\mathrm{NH}_{3}$ one, as it is usually found in other regions. 


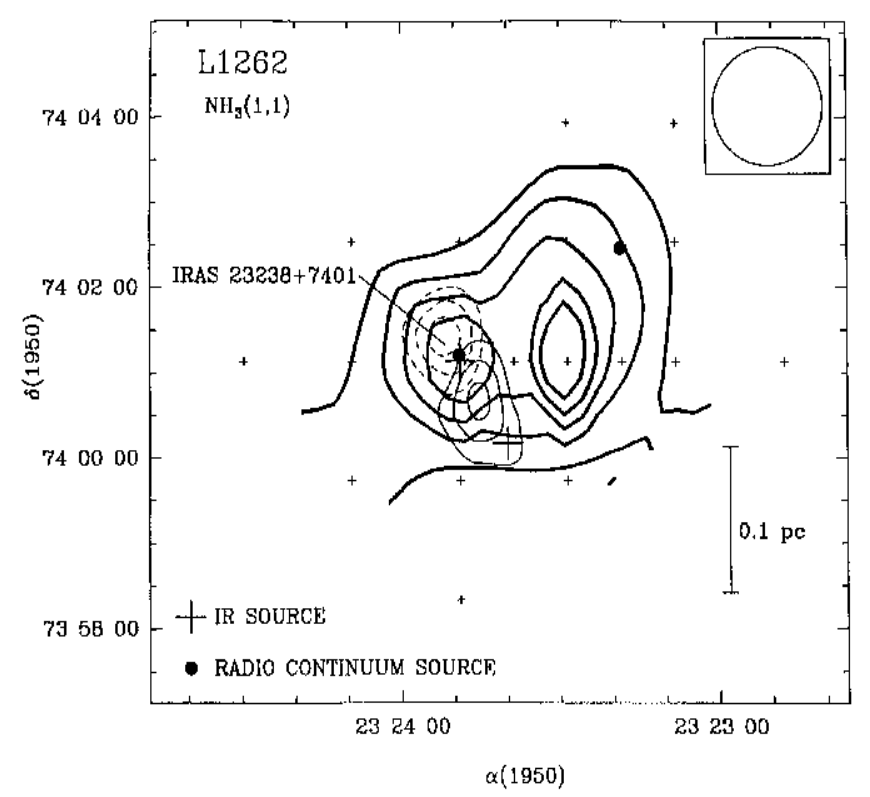

Fig. 20. Same as Fig. 4, for the L1262 region. The ammonia lowest contour level is $0.15 \mathrm{~K}$, and the increment is $0.1 \mathrm{~K}$. The map of the CO outflow is from Yun \& Clemens (1994)

\section{Discussion}

\subsection{Location of the exciting sources of the outflows}

Through the $(J, K)=(1,1)$ and $(2,2)$ inversion transitions of the $\mathrm{NH}_{3}$ molecule we have studied the dense gas in a sample of 15 regions with signs of star formation, as indicated by the presence of outflow activity. We have been able to detect and map the $\mathrm{NH}_{3}$ emission in 14 of these regions. This high rate of detections is a clear indication of the strong association between $\mathrm{NH}_{3}$ emission and outflow activity. This result confirms the young nature of the observed objects, since they appear to be still associated with (and most of them embedded in) the dense gas from where they have been formed.

In all cases where we have mapped the $\mathrm{NH}_{3}$ emission associated with molecular outflows, the ammonia maximum is located near $(<0.1 \mathrm{pc})$ the position of a candidate for driving the outflow. This result gives support to their identification as the outflow exciting sources, following the criterion proposed by Anglada et al. (1989) for such identification. For the sources of our sample that are only associated with optical signs of outflow, the $\mathrm{NH}_{3}$ emission is weak and does not coincide with any known object in the observed field, suggesting that probably none of the nearby objects that have been detected up to now is related to the outflow excitation.

\subsection{Physical parameters of the dense cores}

The sizes of the condensations we have mapped in $\mathrm{NH}_{3}$ range from $\sim 0.1$ to $\sim 0.8 \mathrm{pc}$ (a somewhat larger value of
$2 \mathrm{pc}$ is obtained for the condensation associated with IRAS $20188+3928$, but this value may be overestimated by an order of magnitude, since we have adopted the upper limit for the distance). We find evidence for some elongation in the condensations we mapped (as was noted, in general, by Myers et al. 1991). However, our angular resolution, in general, is not good enough to allow us to further discuss on the morphology of the sources. In particular, we are not able to establish whether or not these condensations play a relevant role in the collimation of the outflows in these regions (as suggested, e.g., by Torrelles et al. 1983). We note, however, the very high degree of elongation of the condensation associated with IRAS $22376+7455$ in L1251, reminiscent of the $\mathrm{NH}_{3}$ structure observed in L1448 (e.g., Anglada et al. 1989). In both cases (L1251 and L1448), several objects are seen in projection towards the elongated $\mathrm{NH}_{3}$ structure. A high angular resolution study of the L1251 region may be relevant in order to investigate a possible fragmentation of the structure. A higher angular resolution study may also be relevant for other sources of our sample that appear unresolved in our present singledish study.

The intrinsic line widths obtained $\left(\sim 1 \mathrm{~km} \mathrm{~s}^{-1}\right.$; see Table 2) are significantly larger than the expected thermal line widths, since the kinetic temperature estimates for these regions give very low values (see Table 3). Only for the region associated with IRAS $20188+3928$ the line widths reach $\sim 2 \mathrm{~km} \mathrm{~s}^{-1}$ (in some positions). This is also the region where we have obtained the highest value for the kinetic temperature, as estimated from our ammonia observations (we note that in many sources we only could obtain upper limits or we were forced to use estimates from CO data, which are less accurate). Despite the uncertainty in the distance, the luminosity of IRAS $20188+3928$ appears to be higher than for most of the objects studied in our sample. Thus, this object appears to produce a larger perturbation in its molecular high density environment than the others objects we have observed.

In part due to our lack of angular resolution, we are not able to measure in detail the velocity gradient in our regions. However, it is remarkable that in the condensation associated with IRAS $22376+7455$ in L1251, our results show the presence of a strong velocity gradient with sudden velocity shifts of up to $\sim 1 \mathrm{~km} \mathrm{~s}^{-1}$ between contiguous positions. A study with high angular resolution of this region appears to be very promising.

The $\mathrm{H}_{2}$ column densities we have obtained are $\sim 10^{22} \mathrm{~cm}^{-2}$ (assuming $\left[\mathrm{NH}_{3} / \mathrm{H}_{2}\right]=10^{-8}$ ), implying mean visual extinctions $\sim 10$ mag. For L483 we have obtained the highest $\mathrm{H}_{2}$ column density $\left(\sim 10^{23} \mathrm{~cm}^{-2}\right.$, corresponding to a visual extinction of $\sim 100 \mathrm{mag}$ ), suggesting that this object is very deeply embedded.

The masses obtained for the observed regions lie typically in the range from 1 to $100 M_{\odot}$. The values derived coincide, in general, with the virial masses within a factor of 3 . This general trend, observed for the regions 
of our sample, suggests that the condensations are near virial equilibrium and that the assumed $\mathrm{NH}_{3}$ abundance is adequate. L483 is the region for which the calculated mass exceeds the virial mass by the largest factor. This could imply that for this source the cloud is still in the process of gravitational collapse. We note here that recently Myers et al. (1995) have detected asymmetric line profiles in this region consistent with infall motion, according to the modeling of Anglada et al. (1987). Although the uncertainties involved are still large, it seems clear that this object is among the youngest sources in our sample, in agreement with the results of Fuller et al. (1995), which classify this as a very young object.

\subsection{Evolutive differences in the outflow sources}

We have detected and mapped the $\mathrm{NH}_{3}$ emission in 12 out of 13 regions with molecular outflow in our sample. Only in one region, L1048 (for which, in fact, no published map of the CO outflow is available), we failed in detecting ammonia emission (Table 1). The $\mathrm{NH}_{3}$ emission is faint ( $T_{\mathrm{MB}} \lesssim 0.5 \mathrm{~K}$; see Table 2$)$ only in 3 regions (RNO $43, \mathrm{HH} 83$ and L100) of the 12 regions associated with molecular outflow we have mapped. On the other hand, in the two regions without molecular outflow ( $\mathrm{HH} 84$ and $\mathrm{HH} 86 / 87 / 88)$, the ammonia emission is very faint. These results tentatively suggest that the ammonia emission tends to be more intense for those sources associated with molecular outflow than for the sources associated with only "optical" signs of outflow (such as jets and HerbigHaro objects).

In order to substantiate this possible relationship between the type of outflow and the intensity of the $\mathrm{NH}_{3}$ emission, we have complemented the sample of regions observed in this paper with the results of other Haystack $\mathrm{NH}_{3}$ observations reported in the literature. We have studied the distribution of the intensity of the $\mathrm{NH}_{3}$ emission, as measured by the main beam brightness temperature towards the outflow exciting source, in this larger sample of regions. We note here that the $\mathrm{NH}_{3}$ brightness temperature is a good measure of the intensity of the $\mathrm{NH}_{3}$ emission only for sources that fill the beam of the telescope. For unresolved sources, a more adequate comparison should be made in terms of the distance corrected flux density of the ammonia emission ("ammonia luminosity"). As we expect that for nearby sources the angular size of the ammonia emission will be, in general, larger than the telescope beam, we have used the main beam brightness temperature to make the comparison, restricting our sample to nearby enough regions. Thus, we have used sources with $D \leq 1 \mathrm{kpc}$, and completed our sample with the data from Torrelles et al. (1983) (9 sources), Anglada et al. (1989) (13 sources), Benson \& Myers (1989) (5 sources, and 2 additional sources observed at Green Bank), Verdes-Montenegro et al. (1989) (3 sources), and Persi et al. (1994) (1 source).
Our final sample is shown in Table 4. It contains a total of 47 sources, with 21 sources associated only with molecular outflow, 19 sources associated both with optical and molecular outflow and 7 sources with only optical outflow. In Fig. 21 we present the distribution of the $\mathrm{NH}_{3}$ main beam brightness temperature towards the position of the proposed outflow exciting source (Table 4) for the three groups of sources. The mean values of the $\mathrm{NH}_{3}$ brightness temperature are $1.7 \mathrm{~K}$ (only molecular outflow), $1.5 \mathrm{~K}$ (optical and molecular outflow) and $0.5 \mathrm{~K}$ (only optical outflow). Despite the relatively small number of sources with only optical outflow, it is clear that these sources tend to present lower values for the $\mathrm{NH}_{3}$ brightness temperature, while for the sources with molecular outflow the distribution is displaced to higher values of the $\mathrm{NH}_{3}$ brightness temperature. We conclude, thus, that the ammonia emission is in general more intense in molecular outflow sources than in sources with optical outflow.

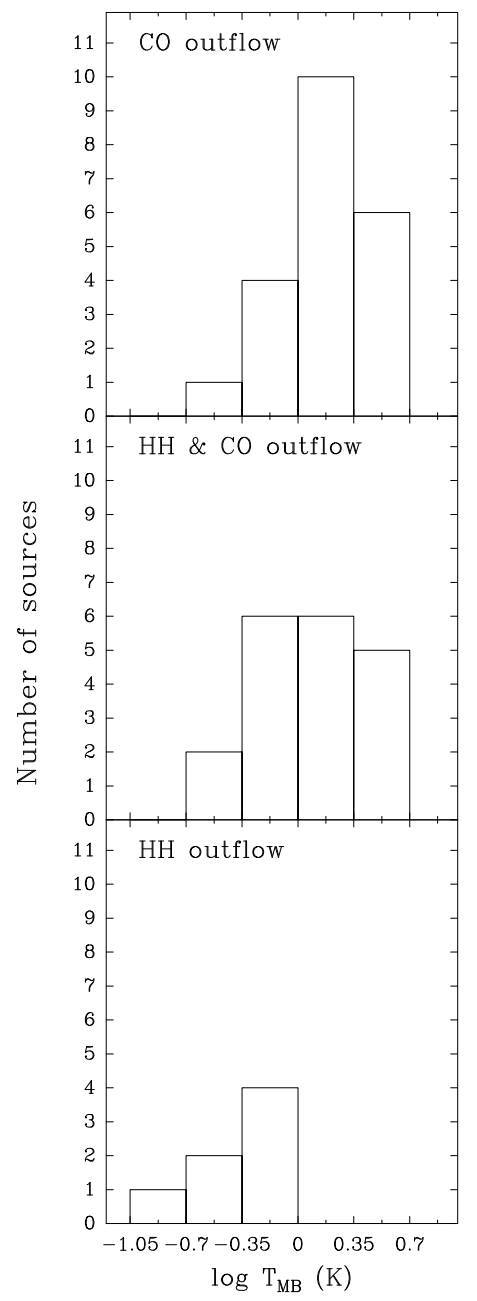

Fig. 21. Distribution of the $\mathrm{NH}_{3}$ main beam brightness temperature for sources with only molecular outflow (top), for sources with both molecular and optical outflow (middle), and for sources with only optical outflow (bottom) 
Table 4. Regions associated with molecular or optical outflow observed in $\mathrm{NH}_{3}{ }^{a}$

\begin{tabular}{|c|c|c|c|c|c|c|c|}
\hline Source & $\begin{array}{c}\text { Outflow } \\
\text { associated }\end{array}$ & Ref. & $\begin{array}{l}T_{\mathrm{MB}} \\
(\mathrm{K})\end{array}$ & $\begin{array}{c}N\left(\mathrm{NH}_{3}\right) \\
\left(10^{14} \mathrm{~cm}^{-2}\right)\end{array}$ & Ref. & $\begin{array}{c}D \\
(\mathrm{pc})\end{array}$ & Ref. \\
\hline L1448 (RNO 13) & $\mathrm{CO}$ & 1 & 0.3 & 0.4 & 2 & 350 & 3 \\
\hline L1448N & $\mathrm{CO}, \mathrm{HH}$ & 4,5 & 3.1 & 4 & 2 & 350 & 3 \\
\hline L1448C & $\mathrm{CO}$ & 4 & 2.5 & 3 & 2 & 350 & 3 \\
\hline GL490 & $\mathrm{CO}$ & 6 & $\leq 0.5$ & $\leq 0.6$ & 7 & 900 & 8 \\
\hline L1455 & $\mathrm{CO}$ & 9 & 2.5 & 1.6 & 2 & 350 & 3 \\
\hline L1489 & $\mathrm{CO}$ & 10 & 0.8 & 4 & 11 & 140 & 8 \\
\hline HH 156 & $\mathrm{HH}$ & 12 & $\leq 0.6$ & $\leq 0.7$ & 11 & 140 & 13 \\
\hline L1524 (Haro 6-10) & $\mathrm{HH}$ & 14 & $\leq 0.6$ & $\leq 1$ & 2 & 140 & 13 \\
\hline L1551 & $\mathrm{CO}, \mathrm{HH}$ & 15,16 & 2.72 & 2.3 & 7 & 140 & 13 \\
\hline HL Tau & $\mathrm{CO}, \mathrm{HH}$ & 17,18 & $\leq 1$ & $\leq 1$ & 7 & 140 & 13 \\
\hline L1527 & $\mathrm{CO}, \mathrm{HH}$ & 19,20 & 2.12 & 5.0 & 11 & 140 & 13 \\
\hline RNO 43 (IRAS 05295+1247) & $\mathrm{CO}, \mathrm{HH}$ & 21,22 & 0.40 & 0.4 & 23 & 400 & 24 \\
\hline HH 83 & $\mathrm{CO}, \mathrm{HH}$ & 25,26 & 0.46 & $0.4-0.5$ & 23 & 470 & 13 \\
\hline HH 84 & $\mathrm{HH}$ & 25 & 0.3 & $0.5-1$ & 23 & 470 & 13 \\
\hline HH $33 / 40$ & $\mathrm{HH}$ & 27 & $\leq 0.3$ & $\leq 0.5$ & 28 & 470 & 13 \\
\hline HH $86 / 87 / 88$ & $\mathrm{HH}$ & 25 & $\leq 0.2$ & $\leq 0.1$ & 23 & 470 & 13 \\
\hline HH 34 & $\mathrm{CO}, \mathrm{HH}$ & 29,30 & 1.3 & $\leq 1$ & 2 & 480 & 31 \\
\hline L1641-N & $\mathrm{CO}$ & 32 & 2.2 & 2.8 & 23 & 480 & 33 \\
\hline HH $38-43$ & $\mathrm{HH}$ & 27 & $\leq 0.5$ & $\leq 0.6$ & 2 & 480 & 31 \\
\hline Haro 4-255 FIR & $\mathrm{CO}$ & 1 & 2.2 & 2.1 & 2 & 480 & 31 \\
\hline B35 & $\mathrm{CO}$ & 10 & 1.2 & 4.0 & 11 & 500 & 34 \\
\hline HH 26 IR & $\mathrm{CO}, \mathrm{HH}$ & 35,36 & 2.7 & 2.1 & 7 & 470 & 13 \\
\hline NGC 2071 & $\mathrm{CO}$ & 37 & 2.44 & 2.2 & 7 & 500 & 8 \\
\hline Mon R2 & $\mathrm{CO}$ & 38 & 1.2 & 1.5 & 7 & 800 & 8 \\
\hline GGD $12-15$ & $\mathrm{CO}$ & 39 & 1.52 & 2.3 & 7 & 1000 & 8 \\
\hline RMon & $\mathrm{CO}, \mathrm{HH}$ & 40,35 & $\leq 0.5$ & $\leq 0.6$ & 7 & 800 & 13 \\
\hline NGC 2264 & $\mathrm{HH}$ & 41 & $\leq 1$ & $\leq 1$ & 2 & 800 & 13 \\
\hline HH 120 & $\mathrm{CO}, \mathrm{HH}$ & 42,43 & $\overline{1} .8$ & $\overline{2} .5$ & 44 & 400 & 45 \\
\hline L43 & $\mathrm{CO}$ & 1 & 2.7 & 2.7 & 2 & 160 & 46 \\
\hline L100 & $\mathrm{CO}$ & 47 & 0.5 & $0.3-0.5$ & 23 & 225 & 48 \\
\hline L483 & $\mathrm{CO}$ & 47 & 4.54 & 14 & 23 & 200 & 49 \\
\hline $\mathrm{R} \mathrm{CrA}$ & $\mathrm{CO}, \mathrm{HH}$ & 1,50 & 2.8 & 2.0 & 2 & 130 & 51 \\
\hline L673 & $\mathrm{CO}$ & 52 & 2.1 & $\geq 2.2$ & 23 & 300 & 3 \\
\hline $\mathrm{HH} 32 \mathrm{a}$ & $\mathrm{CO}, \mathrm{HH}$ & 35,17 & $\leq 0.6$ & $\leq 0.7$ & 2 & 300 & 13 \\
\hline L778 & $\mathrm{CO}$ & 10 & 1.8 & 6.3 & 11 & 250 & 8 \\
\hline B335 & $\mathrm{CO}, \mathrm{HH}$ & 53,54 & 1.2 & 7.9 & 11 & 250 & 13 \\
\hline L1228 & $\mathrm{CO}, \mathrm{HH}$ & 55,56 & 2.71 & 5.3 & 23 & 300 & 56 \\
\hline V1331 Cyg & $\mathrm{CO}$ & 1 & 0.5 & 2.3 & 2 & 700 & 57 \\
\hline L1172 & $\mathrm{CO}$ & 10 & 1.8 & 7.9 & 11 & 440 & 8 \\
\hline NGC 7129 & $\mathrm{CO}, \mathrm{HH}$ & 58,59 & 0.52 & 0.36 & 7 & 1000 & 13 \\
\hline HHL 73 (IRAS 21429+4729) & $\mathrm{CO}$ & 60 & 1.66 & 3.2 & 23 & 900 & 61 \\
\hline HHL 73 (IRAS 21432+4719) & CO, HHL & 60,61 & 1.25 & 1.1 & 28 & 900 & 61 \\
\hline HHL 73 (IRAS 21441+4722) & $\mathrm{CO}$ & 60 & 0.7 & $\geq 0.9$ & 23 & 900 & 61 \\
\hline S140N & $\mathrm{CO}, \mathrm{HH}$ & 31,62 & 0.88 & 1.4 & 28 & 900 & 63 \\
\hline L1251 (IRAS 22343+7501) & $\mathrm{CO}, \mathrm{HH}$ & 64,65 & 0.29 & 1.7 & 23 & 300 & 66 \\
\hline L1251 (IRAS 22376+7455) & $\mathrm{CO}, \mathrm{HH}$ & 64,67 & 1.71 & 3.1 & 23 & 300 & 66 \\
\hline L1262 & $\mathrm{CO}$ & 47 & 1.57 & $\geq 4.9$ & 23 & 200 & 68 \\
\hline
\end{tabular}

${ }^{a}$ Regions with distance $\leq 1 \mathrm{kpc}$.

References: (1) Levreault 1985; (2) Anglada et al. 1989; (3) Herbig \& Jones 1983; (4) Bachiller et al. 1990; (5) Eiroa et al. 1994a; (6) Snell et al. 1984; (7) Torrelles et al. 1983; (8) Fukui et al. 1993; (9) Goldsmith et al. 1984; (10) Myers et al. 1988; (11) Benson \& Myers 1989; (12) Strom et al. 1986; (13) Reipurth 1994; (14) Elias 1978; (15) Snell et al. 1980; (16) Mundt \& Fried 1983; (17) Edwards \& Snell 1982; (18) Mundt et al. 1988; (19) Eiroa et al. 1994a; (20) Heyer et al. 1987; (21) Edwards \& Snell 1984; (22) Jones et al. 1984; (23) this paper; (24) Maddalena \& Morris 1987; (25) Reipurth 1989; (26) Bally et al. 1994; (27) Haro 1953; (28) Verdes-Montenegro et al. 1989; (29) Haro 1959; (30) Chernin \& Masson 1995; (31) Genzel et al. 1981; (32) Fukui et al. 1986; (33) Chen et al. 1993; (34) Felli et al. 1992; (35) Herbig 1974; (36) Snell \& Edwards 1982; (37) Bally 1982; (38) Loren 1981; (39) Rodríguez et al. 1982; (40) Cantó et al. 1981; (41) Adams et al. 1979; (42) Cohen \& Schwartz 1987; (43) Olberg et al. 1989; (44) Persi et al. 1994; (45) Petterson 1984; (46) Chini 1981; (47) Parker et al. 1988; (48) Reipurth \& Gee 1986; (49) Ladd et al. 1991a; (50) Strom et al. 1974; (51) Marraco \& Rydgren 1981; (52) Armstrong \& Winnewisser 1989; (53) Frerking \& Langer 1982; (54) Vrba et al. 1986; (55) Haikala \& Laureijs 1989; (56) Bally et al. 1995; (57) Chavarría-K 1981; (58) Loren 1977; (59) Ray et al. 1990; (60) Dobashi et al. 1993; (61) Gyulbudaghian et al. 1987; (62) Eiroa et al. 1993; (63) Crampton \& Fisher 1974; (64) Sato \& Fukui 1989; (65) Balázs et al. 1992; (66) Kun \& Prusti 1993; (67) Eiroa et al. 1994b; (68) Parker et al. 1991. 
We should note that recent sensitive studies have detected weak $\mathrm{CO}$ outflows in regions where previous studies failed in the detection (e.g., in $\mathrm{HH}$ 1-2 or $\mathrm{HH} 34$; Chernin \& Masson 1995). We have not attempted to take into account the effect of the intensity of the molecular outflow in our study, and we have only considered whether or not an outflow detection has been reported in a given region.

The fact that the sources of molecular outflow present more intense ammonia emission can be interpreted as indicating that these sources are deeply embedded in the high density gas, and surrounded by a larger amount of molecular gas, while those sources with only optical outflow have already dispersed the molecular core or escaped from it. This interpretation can be corroborated by comparing the estimated column density in the sources listed in Table 4. We found that the ammonia column density towards the outflow exciting source decreases as the outflow activity becomes prominent in the optical. The mean values of the $\mathrm{NH}_{3}$ column density are $3.310^{14} \mathrm{~cm}^{-2}$ (only molecular outflow), $2.210^{14} \mathrm{~cm}^{-2}$ (optical and molecular outflow) and $6.610^{13} \mathrm{~cm}^{-2}$ (only optical outflow). In Fig. 22 we show the distribution of the $\mathrm{NH}_{3}$ column density for the three groups of sources. A similar correlation is obtained if the comparison is made in terms of the estimated mass of the associated core, but in this last case it is unclear to what extent should the $\mathrm{NH}_{3}$ emission be considered as associated with a given object.

These results suggest an evolutive sequence of the sources, traced by the intensity of the ammonia emission and the observational appearance of the outflow. Molecular and optical outflow would be phenomena that dominate, observationally, at different stages of the early stellar evolution. In the younger objects molecular outflows will be prominent, while optical outflows will progressively show up as the star evolves. However, this result does not exclude that both phenomena could coexist simultaneously as is required in the so-called "unified models", in which molecular outflows are driven by highly collimated jets (e.g., Raga et al. 1993); only the observational appearance of the outflow evolves in time as the star loses progressively the surrounding high density gas. In this scenario, the driving optical jet is becoming visible as a consequence of the ambient molecular material being progressively removed by the effect of the molecular outflow itself. Alternatively, the observed differences could represent intrinsic differences in the amount of molecular high density gas from one to another region.

\section{Conclusions}

We have detected and mapped the ammonia emission in 14 sources of a sample of 15 sources associated with molecular and/or optical outflows. In addition, we have searched for $\mathrm{H}_{2} \mathrm{O}$ maser emission towards 9 regions, and detected a weak $\mathrm{H}_{2} \mathrm{O}$ maser near IRAS $20188+3928$. Our main

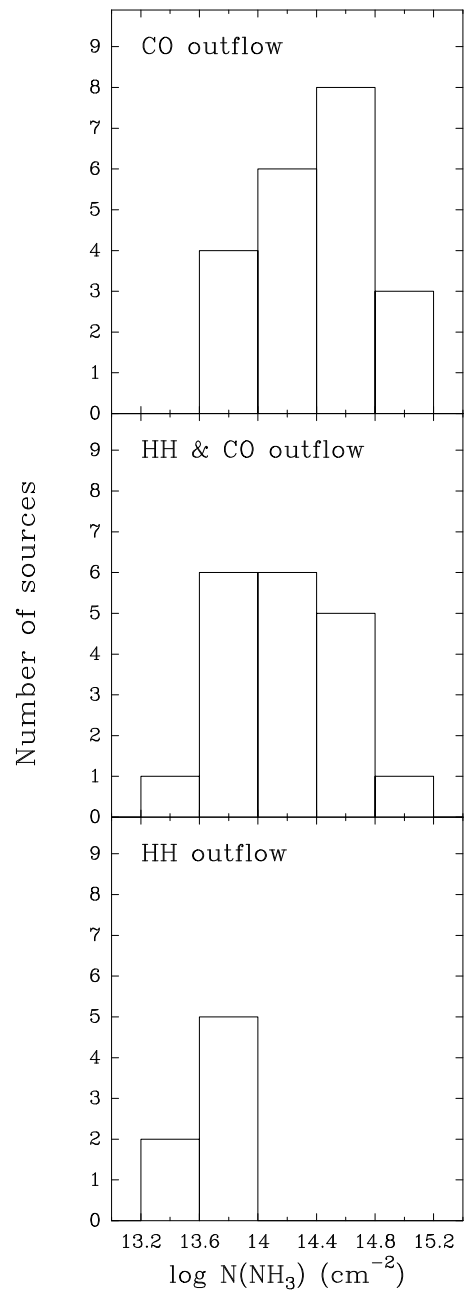

Fig. 22. Distribution of the $\mathrm{NH}_{3}$ column density for sources with only molecular outflow (top), for sources with both molecular and optical outflow (middle), and for sources with only optical outflow (bottom)

conclusions drawn from this study can be summarized as follows:

1. In all the molecular outflow regions we have mapped, the $\mathrm{NH}_{3}$ emission peak is very close to the position of a good candidate for the outflow exciting source.

2. For the two regions with only optical outflow (HH 84 and $\mathrm{HH} 86 / 87 / 88)$, no object is detected towards the observed (weak) $\mathrm{NH}_{3}$ condensations, suggesting a non local origin for the flow excitation.

3. We found that, in general, the $\mathrm{NH}_{3}$ condensations are very cold, with line widths dominated by non thermal (turbulent) motions. Among the observed sources, IRAS $20188+3928$ appears to be the object that produces a larger perturbation in its molecular high density environment. Evidence for disruption of the L1228 $\mathrm{NH}_{3}$ core has also been found.

4. The ammonia condensations appear to be, in general, close to virial equilibrium. L483 is remarkable in 
being associated with the largest visual extinction of our sample and being in a possible gravitational collapse, making this source a very good candidate to be a very young deeply embedded object.

5. A very elongated $\mathrm{NH}_{3}$ structure, apparently associated with several young objects and with strong velocity gradients, has been found near IRAS $22376+7455$ in L1251.

6. Our main result is the discovery of a correlation between the intensity of the $\mathrm{NH}_{3}$ emission and the presence of molecular or optical outflow. The $\mathrm{NH}_{3}$ brightness temperature and column density decrease as the outflow activity becomes prominent in the optical. This result suggests an evolutive scheme in which young objects lose progressively their neighboring high density gas. More $\mathrm{NH}_{3}$ observations towards the exciting sources of optical jets and $\mathrm{HH}$ objects could be very useful to increase the sample in order to allow a more detailed study.

Acknowledgements. We thank the referee, R. Cesaroni, for his careful review of the paper. G.A. and J.F.G. acknowledge support from DGICYT grant PB92-0900, Spain. J.F.G. is also supported by Junta de Andalucía, Spain.

\section{References}

Adams M.T., Strom K.M., Strom S.E., 1979, ApJ 230, L183

André P., Ward-Thompson D., Barsony M., 1993, ApJ 406, 122

Anglada G., Rodríguez L.F., Cantó J., Estalella R., López R., 1987, A\&A 186, 280

Anglada G., Rodríguez L.F., Torrelles J.M., et al., 1989, ApJ 341,208

Anglada G., Rodríguez L.F., Cantó J., Estalella R., Torrelles J.M., 1992, ApJ 395, 494

Anglada G., Estalella R., Mauersberger R., et al., 1995, ApJ 443,682

Anglada G., et al., 1996 (in preparation)

Armstrong J.T., Winnewisser G., 1989, A\&A 210, 373

Bachiller R., Cernicharo J., 1986, A\&A 168, 262

Bachiller R., Cernicharo J., Martín-Pintado J., Tafalla M., Lazareff B., 1990, A\&A 231, 174

Balázs L.G., Eislöffel J., Kelemen J., Kun M., 1992, A\&A 255, 281

Bally J., 1982, ApJ 261, 558

Bally J., Devine D., 1994, ApJ 428, L65

Bally J., Langer W.D., Stark A.A., Wilson R.W., 1987, ApJ $312, \mathrm{~L} 45$

Bally J., Castets A., Duvert G., 1994, ApJ 423, 310

Bally J., Devine D., Fesen R.A., Lane A.P., 1995, ApJ 454, 345

Bence S.J., Richer J.S., Padman R., 1996, MNRAS (in press)

Benson P.J., Myers P.C., 1989, ApJS 71, 89

Benson P.J., Myers P.C., Wright E.L., 1984, ApJ 279, L27

Cabrit S., Goldsmith P.F., Snell R.L., 1988, ApJ 334, 196

Cantó J., Rodríguez L.F., Barral J.F., Carral P., 1981, ApJ 244,102

Chavarría-K C., 1981, A\&A 101, 105

Chen H., Fukui Y., Yang J., 1992, ApJ 398, 544
Chen H., Tokunaga T., Strom K.M., Hodapp K.-W., 1993, ApJ 407, 639

Chen H., Zhao J.H., Ohashi N., 1995, ApJ 450, L71

Chernin L. M., Masson C.L., 1995, ApJ 443, 181

Chini R., 1981, A\&A 99, 346

Cohen M., 1980, AJ 85, 29

Cohen M., Schwartz R.D., 1983, ApJ 265, 877

Cohen M., Schwartz R.D., 1987, ApJ 316, 311

Crampton D., Fisher W.A., 1974, Pub. Dominion Ap. Obs. 14, 283

Curiel S., Raymond J.C., Rodríguez L.F., Cantó J., Moran J.M., 1990, ApJ 365, L85

Dobashi K., Yonekura Y., Mizuno A., Fukui Y., 1992, AJ 104, 1525

Dobashi K., Onishi T., Iwata T., et al., 1993, AJ 105, 1487

Eiroa C., Lenzen R., Miranda L.F., et al., 1993, AJ 106, 613

Eiroa C., Miranda L.F., Anglada G., Estalella R., Torrelles J.M., 1994a, A\&A 283, 973

Eiroa C., Torrelles J.M., Miranda L.F., Anglada G., Estalella R., 1994b, A\&AS 108, 73

Edwards S., Snell R.L., 1982, ApJ 261, 151

Edwards S., Snell R.L., 1984, ApJ 281, 237

Elias J.H., 1978, ApJ 224, 857

Felli M., Palagi F., Tofani G., 1992, A\&A 255, 293

Frerking M.A., Langer W.D., 1982, ApJ 256, 523

Fukui Y., 1989, in Low Mass Star Formation and Pre-Main Sequence Objects, Reipurth B. (ed.). Garching: ESO, p. 95

Fukui Y., Sugitani K., Takaba H., et al., 1986, ApJ 311, L85

Fukui Y., Takaba H., Iwata T., Mizuno A., 1988, ApJ 325, L13

Fukui Y., Iwata T., Mizuno A., Bally J., Lane P., 1993, in Protostars and Planets III, Levy E.H. \& Lunine J.I. (eds.). Tucson: Univ. of Arizona Press, p. 603

Fuller G.A., Myers P.C., 1993, ApJ 418, 273

Fuller G.A., Lada E.A., Masson C.R., Myers P.C., 1995, ApJ 453,754

Genzel R., Reid M.J., Moran J.M., Downes D., 1981, ApJ 286, 599

Goldsmith P.F., Snell R.L., Hemeon-Heyer M., Langer W.D., 1984, ApJ 286, 599

Goodman A.A., Benson P.C., Fuller G.A., Myers P.C., 1993, ApJ 406, 528

Gyulbudaghian A.L., Glushkov Yu.I., Denisyuk E.K., 1978, ApJ 224, L137

Gyulbudaghian A.L., Rodríguez L.F., Mendoza-Torres E., 1987, Rev. Mex. Astron. Astrofis. 15, 53

Haikala L.K., Laureijs R.J., 1989, A\&A 223, 287

Haikala L.F., Miller M., Gierens K., Winnewisser G., 1991, in Molecular Clouds, James R.A. \& Millar T.J. (eds.). Cambridge: Cambridge Univ. Press, p. 25

Harju J., Walmsley C.M., Wouterloot J.G.A., 1991, A\&A 245, 643

Haro G., 1953, ApJ 117, 73

Haro G., 1959 (private communication to G.H. Herbig)

Herbig G.H., 1974, Lick Obs. Bull. No. 658

Herbig G.H., Jones B.F., 1983, AJ 88, 1040

Heyer M.H., Snell R.L., Goldsmith P.F., Myers P.C., 1987, ApJ 321,370

Jones B.F., Cohen M., Sirk M., Jarret R., 1984, AJ 89, 1404

Kun M., Prusti T., 1993, A\&A 272, 235

Lada C.J., 1985, ARA\&A 23, 267

Ladd E.F., Adams F.D., Casey S., et al., 1991a, ApJ 366, 203 
Ladd E.F., Adams F.D., Casey S., et al., 1991b, ApJ 382, 555

Lebrun F., 1986, ApJ 306, 16

Levreault R.M., 1985, Ph.D. Thesis, University of Texas at Austin

Levreault R.M., 1988, ApJS 67, 283

Little L.T., Bergman P., Cunningham C.T., et al., 1988, A\&A 205, 129

Lynds B.T., 1962, ApJS 7, 1

Loren R.B., 1977, ApJ 218, 716

Loren R.B., 1981, ApJ 249, 550

Maddalena R.J., Morris M., 1987, ApJ 323, 179

Maddalena R.J., Morris M., Moscowitz J., Thaddeus P., 1986, ApJ 303, 375

Marraco H.G., Rydgren A.E., 1981, AJ 86, 62

McMullin J.P., Mundy L.G., Blake G.A., 1994, ApJ 137, 305

Molinari S., Liseau R., Lorenzetti D., 1993, A\&AS 101, 59

Morata O., et al., 1996 (in preparation)

Morgan J.A., Bally J., 1991, ApJ 372, 505

Morgan J.A., Snell R.L., Strom K.M., 1990, ApJ 362, 274

Morgan J.A., Schloerb F.P., Snell R.L., Bally J., 1991, ApJ 376,618

Mundt R., Fried J.W., 1983, ApJ 274, L83

Mundt R., Bührke T., Fried J.W., et al., 1984, A\&A 140, 17

Mundt R., Brugel E.W., Bührke T., 1987, ApJ 319, 275

Mundt R., Ray T.P., Bührke T., 1988, ApJ 333, L69

Mundy L.G., McMullin J.P., Grossman A.W., Sandell G., 1993, Icarus 106, 11

Myers P.C., Heyer M., Snell R.L., Goldsmith P.F., 1988, ApJ 324, 907

Myers P.C., Fuller G.A., Goodman A.A., Benson P.J., 1991, ApJ 376, 561

Myers P.C., Bachiller R., Caselli P., et al., 1995, ApJ 449, L65

Nakano M., Sugitani K., Sato F., Ogura K., 1994, ApJ 423, L147

Odenwald S.F., Schwartz P.R., 1993, ApJ 405, 706

Ogura K. 1985, in IAU Symp. 115, Star Forming Regions, Peimbert M. \& Jugaku J. (eds.). Dordrecht: Reidel, p. 341

Ogura K., Walsch J.R., 1991, AJ 101, 185

Olberg M., Reipurth B., Booth R.S., 1989, in The Physics and Chemistry of Interstellar Molecular Clouds, Winnewisser G. \& Armstrong J.T. (eds.) Lect. Notes Phys. 331, 120

Palla F., Brand J., Cesaroni R., Comoretto G., Felli M., 1991, A\&A 246, 249

Parker N.D., 1991, MNRAS 251, 6

Parker N.D., Padman R., Scott P.F., Hills R.E., 1988, MNRAS 234, L67

Parker N.D., Padman, Scott P.F., 1991, MNRAS 252, 442

Pastor J., Estalella R., López R., et al., 1991, A\&A 252, 320

Pauls T.A., Wilson T.L., Bieging J.H., Martin R.N., 1983, A\&A 124, 23

Persi P., Ferrari-Toniolo M., Marenzi A.R., et al., 1994, A\&A 282, 233

Petterson B., 1984, A\&A 117, 183

Pudritz R.E., 1988, in Galactic and Extragalactic Star Formation, Pudritz R.E. \& Fich M. (eds.). Dordrecht: Kluwer, p. 135

Pudritz R.E., Norman C.A., 1983, ApJ 274, 677

Raga A.C., Cantó J., Calvet N., Rodríguez L.F., Torrelles J.M., 1993, A\&A 276, 539
Ray T.P., 1987, A\&A 171, 145

Ray T.P., Poetzel R., Solf J., Mundt R., 1990, ApJ 357, L45

Reipurth B., 1985, A\&AS 61, 319

Reipurth B., 1989, A\&A 220, 249

Reipurth B., 1991, in The Physics of Star Formation and Early Stellar Evolution, Lada C.J. \& Kylafis N.D. (eds.). Dordrecht: Kluwer, p. 497

Reipurth B., 1994, A general catalogue of Herbig-Haro objects, electronically published via anon. ftp to ftp.hq.eso.org, directory /pub/Catalogs/Herbig-Haro

Reipurth B., Gee G., 1986, A\&A 166, 148

Reipurth B., Bally J., Graham J.A., Lane A., Zealey W.J., 1986, A\&A 164, 51

Reipurth B., Chini R., Krugel E., Kreysa E., Sievers A., 1993, A\&A 273,221

Rodríguez L.F., 1990, in The Evolution of the Interstellar Medium, Blitz L. (ed.), ASP Conf. Ser. 12, 183

Rodríguez L.F., Carral P., Ho P.T.P., Moran J.M., 1982, ApJ 260, 635

Rodríguez L.F., Reipurth B., 1994, A\&A 281, 882

Sato F., Fukui Y., 1989, ApJ 343, 773

Sato F., Mizuno A., Nagahama T., et al., 1994, ApJ 435, 279

Schwartz P.R., Gee G., Huang Y.-L., 1988, ApJ 327, 350

Snell R.L., Edwards S., 1982, ApJ 259, 668

Snell R.L., Loren R.B., Plambeck R.L., 1980, ApJ 239, L17

Snell R.L., Scoville N.Z., Sanders D.B., Erickson N.R., 1984, ApJ 284, 176

Smith H.A., Fischer J., Mozurkewich D., Schwartz P., 1989, Bull. AAS 20, 1093

Strom S.E., Grasdalen G.L., Strom K.M., 1974, ApJ 191, 111

Strom K.M., Strom S.E., Wolff S.C., Morgan J., Wenz M., 1986, ApJS 62, 39

Strom K.M., Newton G., Strom S.E., et al., 1989a, ApJS 71, 183

Strom K.M., Margulis M., Strom S.E., 1989b, ApJ 345, L79

Strom K.M., Margulis M., Strom S.E., 1989c, ApJ 346, L33

Tafalla M., Myers P.C., Wilner D.J., 1994, in Clouds, Cores and Low Mass Stars, Clemens D. and Barvainis R. (eds.) ASP Conf. Ser. 65, 391

Takaba H., Fukui Y., Fujimoto Y., et al., 1986, A\&A 166, 276

Terebey S., Vogel S.N., Myers P.C., 1989, ApJ 340, 472

Torrelles J.M., Rodríguez L.F., Cantó J., et al., 1983, ApJ 274, 214

Uchida Y., Shibata K., 1985, PASJ 37, 515

Ungerechts H., Walmsley C.M., Winnewisser G., 1986, A\&A 157,207

Verdes-Montenegro L., Torrelles J.M., Rodríguez L.F., et al., 1989, ApJ 346, 193

Vrba F.J., Luginbuhl C.B., Strom S.E., Strom K.M., Heyer M.H., 1986, AJ 92, 633

Weintraub D.A., 1990, ApJS 74, 575

Wilking B.A., Mundy L.G., Blackwell J.H., Howe J.E., 1989, ApJ 345, 257

Wilking B.A., Blackwell J.H., Mundy L.G., 1990, AJ 100, 758

Wilking B.A., Claussen M.J., Benson P.J., et al., 1994, ApJ 431, L119

Xiang D., Turner B.E., 1995, ApJS 99, 121

Yun J.L., Clemens D.P., 1994, ApJS 92, 145

Zhou S., Wu Y., Evans N.J., et al., 1989, ApJ 346, 168 